\title{
Article \\ A BEM-based actuator disk model for wind turbine wakes considering atmospheric stability
}

\author{
Xing Xing Han ${ }^{1}{ }^{1}$, De You Liu ${ }^{1}$, Chang $\mathrm{Xu}^{2, *}$, Wen Zhong Shen ${ }^{3}$, Lin Min $\mathrm{Li}^{2}$ and Fei Fei Xue ${ }^{1}$ \\ 1 College of Water Conservancy and Hydropower Engineering, Hohai University, China; hantone@hhu.edu.cn \\ (Xing Xing Han), Liudyhhuc@163.com (De You Liu), xuefeifeihhu@163.com(Fei Fei Xue) \\ 2 College of Energy and Electrical Engineering, Hohai University, China; lilinmin@hhu.edu.cn (Lin Min Li) \\ 3 Department of Wind Energy, Technical University of Denmark, Denmark; wzsh@dtu.dk \\ * Correspondence: zhuifengxu@hhu.edu.cn;
}

\begin{abstract}
Atmospheric stability affects wind turbine wakes significantly. High-fidelity approaches such as large eddy simulations (LES) with the actuator line (AL) model which predicts detailed wake structures, fail to be applied in wind farm engineering applications due to its expensive cost. In order to make wind farm simulations computationally affordable, this paper proposes a new actuator disk model (AD) based on the blade element method (BEM) and combined with Reynolds-averaged Navier-Stokes equations (RANS) to model turbine wakes under different atmospheric stability conditions. In the proposed model, the upstream reference velocity is firstly estimated from the disk averaged velocity based on the one-dimensional momentum theory, and then is used to evaluate the rotor speed to calculate blade element forces. Flow similarity functions based on field measurement are applied to limit wind shear under strongly stable conditions, and turbulence source terms are added to take the buoyant-driven effects into consideration. Results from the new AD model are compared with field measurements and results from the AD model based on the thrust coefficient, the BEM-AD model with classical similarity functions and a high-fidelity LES approach. The results show that the proposed method is better in simulating wakes under various atmospheric stability conditions than the other AD models and has a similar performance to the high-fidelity LES approach however in much lower computational cost.
\end{abstract}

Keywords: wind turbine; wake; atmospheric stability; actuator disk; BEM

\section{Introduction}

As a measure of turbulence exchanges in the atmospheric surface layer (ASL), atmospheric stability can significantly affect the wind turbine wake deficit and its recovery rate. In general, turbulence exchanges between the wake and the atmosphere are depressed under stable conditions. High wake deficits and slow wake recovery thus are usually observed in the stable stratification boundary layer [1,2]. Since wakes play critical roles in wind farm energy production and the fatigue loads of wind turbines, there is an increasing interest in studying effects of atmospheric stability on wakes and developing wake models considering atmospheric stability.

The impact of atmospheric stability on wakes are widely observed in wind tunnel measurements [3-5] and field experiments [1,2,6,7]. According to the wind tunnel measurements in Chamorror et al. [3], the stronger inlet wind shear in the stable case leads to a slightly stronger turbulence intensity and extends the region of enhanced turbulence intensity from a distance of about 4-5.5 rotor diameters to 3 and 6 rotor diameters downwind of the turbine location. In Zhang et al. [4], a 15\% smaller velocity deficit at the wake center, a more rapid momentum recovery due to an enhanced radial momentum transport, a $20 \%$ higher peak turbulence intensity were observed in the unstable case, as compared to 

Han et al. [1] found that the velocity deficit decreases more slowly under stable conditions and more quickly under unstable conditions. According to the field experiments of wakes using lidars [2,7] or masts [1], the double-bell near-wake shape was clearly observed in very stable cases. Measurements showed the strong dependence of the vertical wake propagation on the atmospheric stability [2,7]: the wake tends to follow the terrain down the ridge with a maximum inclination of $-28^{\circ}$ in very stable cases or be advected upwards by up to $29^{\circ}$ above the horizontal plane in unstable cases. Those observations suggest that atmospheric stability should be considered for improved wake models and predictions of wind power harvesting.

In the current stage, high-fidelity large eddy simulations with the actuator line model (AL-LES) $[8,9]$ or the actuator disk model (AD-LES) $[2,10]$ are widely used to study the structure and dynamics of wind turbine wakes in varying stability cases. "Precursor" atmospheric simulations of the thermal stratified layer are usually required to provide ambient conditions applied in the simulations of wind turbine wakes [2,11]. Energy equations are modeled while buoyancy and Coriolis force due to planetary rotation are added in the momentum transport equations. There are no special corrections of turbulence terms for wake modeling as compared to that in RANS. As Peña [12] argued, proper characterizations of the shifts in length and velocity scales of the turbulence are required to model a non-neutral atmosphere, which results in the special treatment of the turbulence Prandtl number in the Smagorinsky subgrid-scale model of the Simulator fOr Wind Farm Applications (SOWFA) [11]. The LES approaches allow to capture the near wake structure, resolve the interactions of tip vortices with large-scale eddies of the ambient flow and wake meandering. Fair agreements between measurements and the LES predictions were presented in literature $[2,13]$. However, the high-fidelity approaches are computational expensive for wind energy engineering applications. In the AL-LES modeling of rotors, the tip of blade is not allowed to travel more than one mesh cell over a time step [14]. Noting the high resolution mesh in the AL simulations to capture the detail wake structure, this limitation of AL-LES method results in a very small time step (about $0.01 \mathrm{~s}$ ). Although an AD-LES method with a coarse spatial resolution could bring a relaxed time step and make large-scale wind farm simulations computationally affordable for research purposes, the high-fidelity approaches still have a long way for engineering applications.

An order-reduced alternative to the LES approach is the AD model based on the thrust coefficient (CT-AD) or based on BEM (BEM-AD) in transient RANS with a large time step (about $1 \mathrm{~s}$ ) or even in steady RANS. Classical CT-AD approachs combined with turbulence corrections such as the El Kasmi model [15] and the $k-\varepsilon-f_{p}$ model [16] are now commonly used in wake modeling [15-18] and validated by numerous experiments $[3,19,20]$ under neutral conditions. Recently, some BEM-AD models $[10,18]$ that take into account effects of the turbine blade induced flow rotation are also introduced in RANS. Using BEM to distribute the blade force through the rotor provides an applicable way to model wakes in yaw or pitch conditions and allows to capture the near wake more precisely than the CT-AD. In non-neutral cases, the inlet boundary conditions are imposed with similarity functions of atmospheric stability and special turbulence terms are added to model effects of atmospheric stability on turbulence $[1,21]$. Model uncertainties might be raised due to the model ability to keep flow homogeneity and the overestimated wind shear by the classical similarity functions. A widely used turbulence model for the thermal stratified boundary layer was developed by Alinot and Masson [22] by calibrating the coefficient of the buoyant terms in the $\varepsilon$ transport equation. However, van der Laan et al. [21] showed that this model cannot keep the flow homogeneity in large domain under unstable conditions and thus developed a turbulence model consistent with the classical similarity functions. In general, the height of surface boundary layer decreases with atmospheric stability down to $10 \mathrm{~m}$ in very stable cases. The decreased boundary layer height limits the wind shear and results in a fair lower wind shear than the one based on classical similarity functions [1,23]. In Han et al. [1], the unlimited wind shear predicted by the classical similarity functions was observed in overestimating wind speedup for upslow flow and underestimating it for downslope flow. Consequently, a new set 
of similarity functions based on field measurements in the full range of the Richardson number is estimated to limit the wind shear in very stable conditions [1]. Han et al. [1] thus proposed the FullyRF turbulence model by extending the Laan model with those observed similarity functions.

In this paper, a BEM-based AD model by using the FullyRF turbulence model [1] is developed to simulate turbine wakes under various atmospheric stability for engineering applications. This paper mainly focuses on how the distributed blade force using BEM helps the predictions of near wake structures and the side effects of applying wind profiles with unlimited wind shear in the classical similarity functions on wake modeling. Before discussing the simulation results and measurements of wakes under varying stability conditions (Section 4), we will start with a description of the BEM-AD method and the associated turbulence model in the next section. A detail introduction of simulation cases including computational domain, meshing and boundary conditions is presented in Section 3. Finally, in Section 5 the results of wake simulations are concluded.

\section{Methods}

In the modeling of wind turbine wakes under different stability conditions, the following continuity, momentum and energy equations [24] are solved:

$$
\begin{gathered}
\frac{\partial}{\partial x_{i}}\left(\rho U_{i}\right)=0 \\
\frac{\partial}{\partial t}\left(\rho U_{i}\right)+\frac{\partial}{\partial x_{j}}\left(\rho U_{i} U_{j}\right)=-\frac{\partial p}{\partial x_{i}}+\frac{\partial}{\partial x_{j}}\left[\left(\mu+\mu_{t}\right)\left(\frac{\partial U_{i}}{\partial x_{j}}+\frac{\partial U_{j}}{\partial x_{i}}\right)\right]+S_{u, i} \\
\frac{\partial}{\partial t}(\rho \Theta)+\frac{\partial}{\partial x_{i}}\left(\rho U_{i} \Theta\right)=\frac{\partial}{\partial x_{i}}\left[\left(\mu+\frac{\mu_{t}}{\sigma_{\theta}}\right) \frac{\partial \Theta}{\partial x_{i}}\right]
\end{gathered}
$$

where $\rho$ is the air density, $U_{i}$ is the velocity component in the $x_{i}$ direction, $p$ is the air pressure, $\mu$ is the laminar viscosity, $\mu_{t}$ is the turbulent viscosity, $S_{u, i}$ is the momentum term source imposed by the wind turbine rotor in the $x_{i}$ direction, $\Theta$ is the potential temperature and $\sigma_{\theta}$ is the turbulent Prandtl number.

\subsection{Modeling of Wind Turbine}

\subsubsection{Actuator Disk Model Based on Thrust Coefficient (CT-AD)}

In the origin actuator disk model, the momentum source term in the $x_{i}$ direction due to the thrust is uniformly distributed through the rotor:

$$
S_{u, i}=-\frac{T}{V_{\mathrm{disk}}} \frac{U_{\mathrm{ref}, i}}{U_{\mathrm{ref}}}=-\frac{\rho C_{T} U_{\mathrm{ref}} U_{\mathrm{ref}, i}}{2 \Delta l}
$$

where $T$ is the thrust, $V_{\text {disk }}$ is the disk volume, $C_{T}$ is the thrust coefficient, $U_{\text {ref }}$ is the upstream reference velocity at hub height, $U_{\text {ref, } i}$ is the component velocity $U_{\text {ref }}$ of in $x_{i}$ direction, $\Delta l$ is the disk depth and the negative sign represents the drag effects of thrust on the flow.

For the upstream flow disturbed by a complex terrain or wind turbine wakes, $U_{\text {ref }}$ is unknown and difficult to be evaluated from the local flow directly. According to the one-dimensional momentum theory and ignoring the wind shear, the reference velocity is a function of the rotor-averaged velocity $U_{\text {disk }}$ :

$$
U_{\text {disk }}=\left(1-a_{B}\right) U_{\text {ref }}
$$

in which the induced factor $a_{B}$ [25] is related to the thrust coefficient $C_{T}$ by

$$
a_{B}= \begin{cases}\frac{1}{2}\left(1-\sqrt{1-C_{T}}\right), & C_{T} \leq \frac{8}{9} \\ \frac{C_{T}-4 a_{c}^{2}}{4\left(1-2 a_{c}\right)}, & C_{T}>\frac{8}{9}\end{cases}
$$


where $a_{c}=1 / 3$.

In simulations, the disk-averaged velocity $U_{\text {disk }}$ is calculated by averaging the local velocity in the disk region, then is applied to estimate the upstream reference velocity $U_{\text {ref }}$ based on Equations (5) and (6) and the momentum source $S_{u, i}$ based on Equation (4), respectively.

\subsubsection{Actuator Disk Model Based on Blade Element Method (BEM-AD)}

In the BEM-based actuator disk model, the rotor plane consists of $N$ actuator lines and each of the actuator lines is splitted into $M$ element sections (Fig. 1). The element section collects the local velocity and the rotor speed $\Omega$ to calculate the element force and applies this force in the neighbor cells of the element section. The reference velocity is firstly assessed from the disk-averaged velocity and then is applied to evaluate the rotor speed.

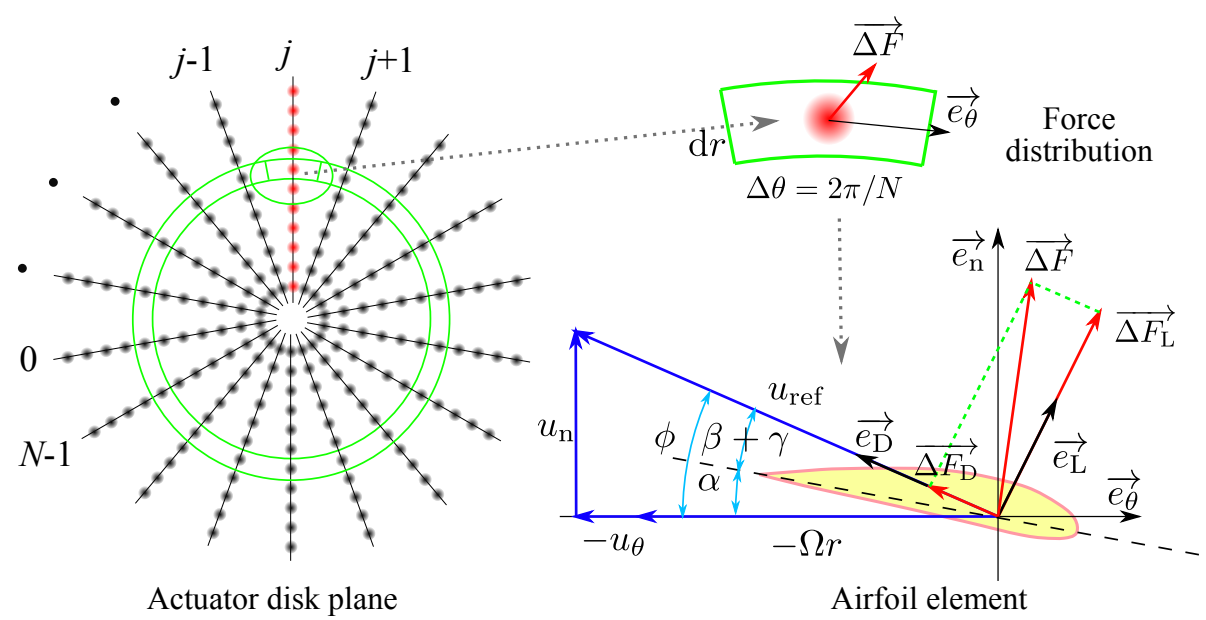

Figure 1. Schemtaics of the BEM-based acutor disk model.

By transforming the local velocity at the blade element into polar velocity components $\left(u_{r}, u_{\theta}\right.$, $\left.u_{n}\right)$, the force of the blade element is:

$$
\overrightarrow{\Delta F}=\frac{B \Delta \theta}{4 \pi} \rho u_{r e l}^{2} c\left(C_{L} \overrightarrow{e_{L}}+C_{D} \overrightarrow{e_{D}}\right) \Delta r
$$

where $B$ is the number of the blades and $c$ is the chord length, $\Delta r$ is the length of the element section. The drag coefficient of the element section, $C_{D}$ and its lift coefficient $C_{L}$ which are functions of the attack anlge $\alpha$, are estimated from XFOIL [26] and then corrected by three-dimensional rotational effects of the blades based on Du et al. [27]. According to Fig. $1, \alpha=\phi-(\beta+\gamma)$ where $\phi=\arctan \left[u_{\mathrm{n}} /\left(\Omega r+u_{\theta}\right)\right]$ is the flow angle, $\beta$ is the blade installation anlge and $\gamma$ is the pitch angle.

The element force is distributed across neighbor cells. The force added to a cell is calculated by:

$$
\overrightarrow{\Delta F_{\text {cell }}}=\sum_{i}^{N \cdot M} \frac{1}{s^{3} \pi^{3 / 2}} \exp \left(-\frac{s_{i}^{2}}{s^{2}}\right) \overrightarrow{\Delta F}_{i} F_{\text {tip }} F_{\text {hub }} \Delta V_{\text {cell }}
$$

where $s_{i}$ the distance of the $i$-th element to the cell and $s$ is the cut-off length scale that takes a value between 2 and 3 cell sizes [28]. $F_{\text {tip }}$ and $F_{\text {hub }}$ are the Prandtl tip loss and hub loss functions [29]:

$$
\begin{gathered}
F_{\text {tip }}=\frac{2}{\pi} \arccos \left[\exp \left(\frac{B(R-r)}{2 r \sin \phi}\right)\right] \\
F_{\text {hub }}=\frac{2}{\pi} \arccos \left[\exp \left(\frac{B\left(r-R_{\text {hub }}\right)}{2 r \sin \phi}\right)\right]
\end{gathered}
$$

where $R$ is the rotor radius, $R_{\text {hub }}$ is the hub radius, $r$ is the radial distance of the element to the rotor center. 


\subsection{Turbulence modeling}

To model effects of atmospheric stability on wind turbine wakes, the extended $k-\varepsilon$ model proposed by Han [1] is applied, in which the eddy viscosity is calibrated with atmospheric stability:

$$
\mu_{t}=\frac{\phi_{\varepsilon}}{\phi_{m} \phi_{k}^{2}} \cdot C_{\mu} \frac{k^{2}}{\varepsilon}
$$

where $C_{\mu}=0.033, k$ and $\varepsilon$ are the turbulence kinetic energy (TKE) and its dissipation (TKD), respectively. $\phi_{m} \equiv \frac{k z}{u_{*}} \frac{\partial U}{\partial z}$ is the dimensionless gradient of wind speed, $\phi_{k} \equiv \sqrt{C_{\mu}} k / u_{*}^{2}$ is the dimensionless TKE and $\phi_{\varepsilon} \equiv \kappa z \varepsilon / u_{*}^{3}$ is the dimensionless TKD where $\kappa=0.4$, the friction speed $u_{*}=\left({\overline{u^{\prime} w^{\prime}}}^{2}+{\overline{v^{\prime} w^{\prime}}}^{2}\right)^{1 / 4}, u^{\prime}, v^{\prime}$ and $w^{\prime}$ are the fluctuations of the longitudinal, lateral and vertical velocity compoments. The dimensionless gradient of potential temperature, $\phi_{h}$ also can be defined as $\frac{\kappa z}{\theta_{*}} \frac{\partial \Theta}{\partial z}$ where the scaling temperature $\theta_{*}=-\overline{w^{\prime} \theta^{\prime}} / u_{*}$ and $\theta^{\prime}$ is the fluctuation of potential temperature.

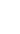

In the extended approach, the turbulence kinetic energy $k$ and its dissipation $\varepsilon$ are modeled by

$$
\begin{gathered}
\frac{\partial}{\partial t}(\rho k)+\frac{\partial}{\partial x_{i}}\left(\rho U_{i} k\right)=\frac{\partial}{\partial x_{j}}\left[\left(\mu+\frac{\mu_{t}}{\sigma_{k}}\right) \frac{\partial k}{\partial x_{j}}\right]+\mathcal{P}+\mathcal{B}-\varepsilon-S_{k, \mathrm{SBL}} \\
\frac{\partial}{\partial t}(\rho \varepsilon)+\frac{\partial}{\partial x_{i}}\left(\rho U_{i} \varepsilon\right)=\frac{\partial}{\partial x_{j}}\left[\left(\mu+\frac{\mu_{t}}{\sigma_{\varepsilon}}\right) \frac{\partial \varepsilon}{\partial x_{j}}\right]+\left(C_{\varepsilon 1} \mathcal{P}-C_{\varepsilon 2} \varepsilon\right) \frac{\varepsilon}{k}+S_{\varepsilon, \mathrm{ABL}}+S_{\varepsilon, \text { wake }}
\end{gathered}
$$

where $\sigma_{k}=1.0, \sigma_{\varepsilon}=1.3, C_{\varepsilon 2}=1.92$, and $C_{\varepsilon 1}=1.24$. $\mathcal{P}$ and $\mathcal{B}$ are the TKE source production due to shear and buoyancy:

$$
\begin{gathered}
\mathcal{P}=\mu_{t}\left(\frac{\partial U_{i}}{\partial x_{j}}+\frac{\partial U_{j}}{\partial x_{i}}\right) \frac{\partial U_{j}}{\partial x_{i}} \\
\mathcal{B}=-\frac{g_{i}}{\Theta_{0}} \frac{\mu_{t}}{\sigma_{\theta}} \frac{\partial \Theta}{\partial x_{i}}
\end{gathered}
$$

where $\Theta_{0}$ is the potential temperature on the ground.

The source term $S_{\varepsilon, \text { wake }}$ is applied to correct the fast wake recovery of the standard $k-\varepsilon$ model [15]:

$$
S_{\varepsilon, \text { wake }}=C_{4 \varepsilon} \frac{\mathcal{P}^{2}}{\rho k}
$$

where $C_{4 \varepsilon}=0.37$.

The TKE source term $S_{\varepsilon, \mathrm{ABL}}$ is applied to maintain flow velocity profiles under non-neutral conditions over a flat ground:

$$
S_{k, \mathrm{ABL}}=\left\{\phi_{m}-\phi_{\varepsilon}-\zeta+\frac{C_{\mu}^{-1 / 2} \kappa^{2}}{\sigma_{k}} \frac{\zeta^{2}}{\phi_{m}}\left[\phi_{k}^{\prime \prime}-\frac{\phi_{k}^{\prime} \phi_{m}^{\prime}}{\phi_{m}}+\frac{\phi_{k}^{\prime}}{\zeta}\right]\right\} \frac{u_{*}^{3}}{\kappa z}
$$

where $\phi^{\prime}=\partial \phi / \partial \zeta, \phi^{\prime \prime}=\partial^{2} \phi / \partial \zeta^{2}$ for $\phi \in\left\{\phi_{k}, \phi_{m}, \phi_{\varepsilon}\right\}$. The TKD source term due to buoyancy, $S_{\varepsilon, \mathrm{ABL}}$ is modelled by:

$$
S_{\varepsilon, \mathrm{ABL}}=C_{\varepsilon 3} \mathcal{B} \frac{\varepsilon}{k}
$$

in which

$$
C_{\varepsilon 3}=\frac{C_{\varepsilon 1} \phi_{m}-C_{\varepsilon 2} \phi_{\varepsilon}}{\zeta}+\frac{C_{\mu}^{-1 / 2} \kappa^{2}}{\sigma_{\varepsilon}} \frac{\phi_{k}}{\phi_{m}}\left[\frac{\zeta \phi_{\varepsilon}^{\prime \prime}}{\phi_{\varepsilon}}-\frac{\zeta \phi_{\varepsilon}^{\prime} \phi_{m}^{\prime}}{\phi_{\varepsilon} \phi_{m}}-\frac{\phi_{\varepsilon}^{\prime}}{\phi_{\varepsilon}}+\frac{\phi_{m}^{\prime}}{\phi_{m}}+\frac{1}{\zeta}\right]
$$

According to the Monin-Obukhov similarity theory [30], $\phi_{m}, \phi_{h}, \phi_{\varepsilon}$ and $\phi_{k}$ are functions of the stability parameter $\zeta=z / L$ where $L$ is the Obukhov length: 


$$
L \equiv-\frac{u_{*}^{3}}{\kappa \frac{g}{\Theta} \overline{w^{\prime} \theta^{\prime}}}
$$

Based on measurements of flows over flat terrain in the ASL, the classical similarity functions commonly used in literature are given as [31,32]:

$$
\begin{gathered}
\phi_{m, \mathrm{cls}}(\zeta)= \begin{cases}\left(1-\gamma_{m} \zeta\right)^{-1 / 4} & -2<\zeta<0 \\
1+\beta_{m} \zeta & 0<\zeta<1\end{cases} \\
\phi_{h, \mathrm{cls}}(\zeta)= \begin{cases}\chi\left(1-\gamma_{h} \zeta\right)^{-1 / 2} & -2<\zeta<0 \\
\chi+\beta_{h} \zeta & 0<\zeta<1\end{cases} \\
\phi_{\varepsilon, \mathrm{cls}}(\zeta)= \begin{cases}1-\zeta & \zeta<0 \\
\phi_{m, \mathrm{cls}}-\zeta & \zeta>0\end{cases} \\
\phi_{k, \mathrm{cls}}(\zeta)=\sqrt{\frac{\phi_{\varepsilon, \mathrm{cls}}(\zeta)}{\phi_{m, \mathrm{cls}}(\zeta)}}
\end{gathered}
$$

where $\gamma_{m}=\gamma_{h}=16, \beta_{m}=\beta_{h}=5$ [32] and $\chi=0.9$.

According to Grachev et al. [33], the applicability of the Monin-Obukhov similarity theory is only valid for the flux Richardson number $R_{f}$ below 0.25 . In the full range of $R_{f}$, the similarity functions based on a field measurement reads [1]:

$$
\begin{gathered}
\phi_{m, \exp }(\zeta)= \begin{cases}\frac{2+100 \zeta^{2}}{2+200 \zeta^{2}} & \zeta<0 \\
(1+6 \zeta)^{\frac{1+16 \zeta^{2}}{1+40 \zeta^{2}}} & \zeta>0\end{cases} \\
\sigma_{\theta, \exp }(\zeta)= \begin{cases}0.9(1-2 \zeta)^{-1 / 2} & \zeta<0 \\
0.9(1+6 \zeta)^{-1 / 8} & \zeta>0\end{cases}
\end{gathered}
$$

where $\sigma_{\theta, \exp }(\zeta)=\phi_{h, \exp }(\zeta) / \phi_{m, \exp }(\zeta)$

Eqs. (17) and (19) can be adapted with the classical similarity functions (the Laan model [21]) or the similarity functions in the full range of $R_{f}$ (the FullyRF model [1]).

Table 1. Turbulence model for non-neutral conditions.

\begin{tabular}{cc}
\hline Turbulence Model & Similarity functions \\
\hline Laan & classical \\
FullyRF & $\phi_{m, \exp }, \phi_{h, \exp }, \phi_{k, \mathrm{cls}}, \phi_{\varepsilon, \mathrm{cls}}$ \\
\hline
\end{tabular}

\section{Simulation Details}

\subsection{The H93-2.0MW Turbine Test Case}

Since the FullyRF similarity functions are derived from field measurements in the Jingbian wind farm where measurements of wind turbine wakes were also carried out [34], the wake models are firstly assessed in this area. In the experimental campaign, two masts numbered M1 and M3 are installed near a H93-2.0MW wind turbine (\#14) to capture the wake profiles. On the masts, cup anemometers were installed at $30 \mathrm{~m}, 50 \mathrm{~m}$ and $70 \mathrm{~m}$. Measurements of the sonic anemometer installed on M1 at $30 \mathrm{~m}$ were applied to estimate the Obukhov length $L$. Three cases (1) M1 $\rightarrow \# 14 \rightarrow$ M3, (2) M3 $\rightarrow \# 14 \rightarrow$ M1 and (3) \#14 $\rightarrow$ M1 are studied with longitudinal distances to the upstream wind turbine rotors of $1.25 D, 2.15 D$ and $5 D$ where $D$ is the rotor diameter. As M3 is near \#14 in case (1) and the terrain 
slope in the wake of cases (2) and (3) is below $2^{\circ}$, the wake is expected to follow the terrain. This is especially for some stable cases as observed in [7]. The average wind direction misalignment at M3 and \#14 due to the complex terrain that is about $2.05^{\circ}$ in unstable cases, $3.75^{\circ}$ in neutral cases and $3.94^{\circ}$ in stable cases, is also applied to correct the inflow wind direction at M3. Since the flow tends to follow the terrain under stable conditions, this misalignment of wind direction is enhanced by atmospheric stability. Taking these terrain effects into considerations, simulations of wakes of the H93-2.0MW wind turbine in flat terrain are expected to be able compared with measurements.

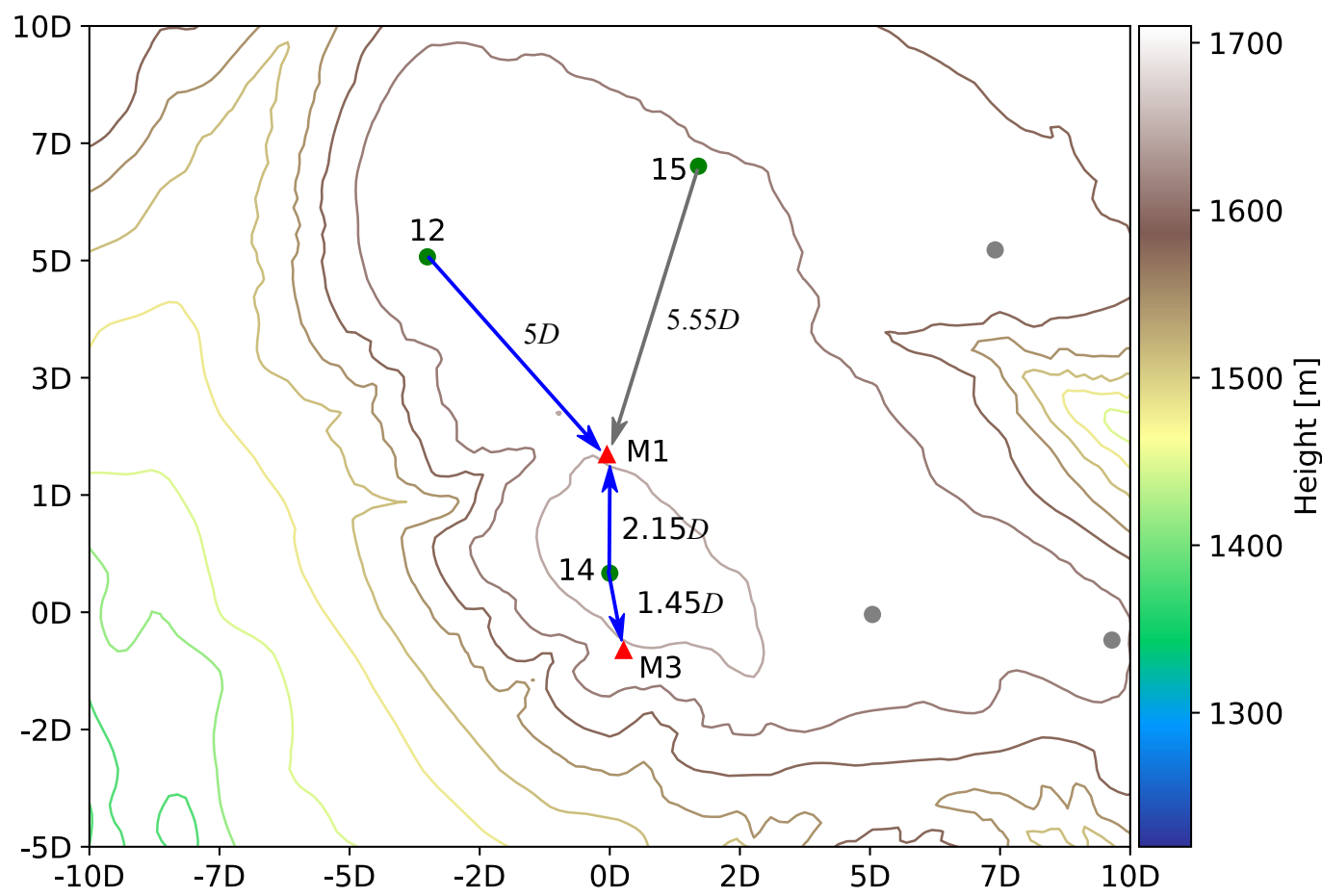

Figure 2. Complex terrain around the H93-2.0MW wind turbine (\#14).

The power curves and thrust coefficient curves of the H93-2.0MW turbine are shown in Fig. 3. Results of BEM are compared with manufacture curves, measurements and other CFD results. The observed power curve and the thrust coefficient curve measured from the strain gauges installed at the bottom of the tower [34] show good agreement with the performance curves from BEM. The large discrepancy of the thrust coefficient from the manufacture and from the BEM calculations are probably due to different implementations of BEM. 


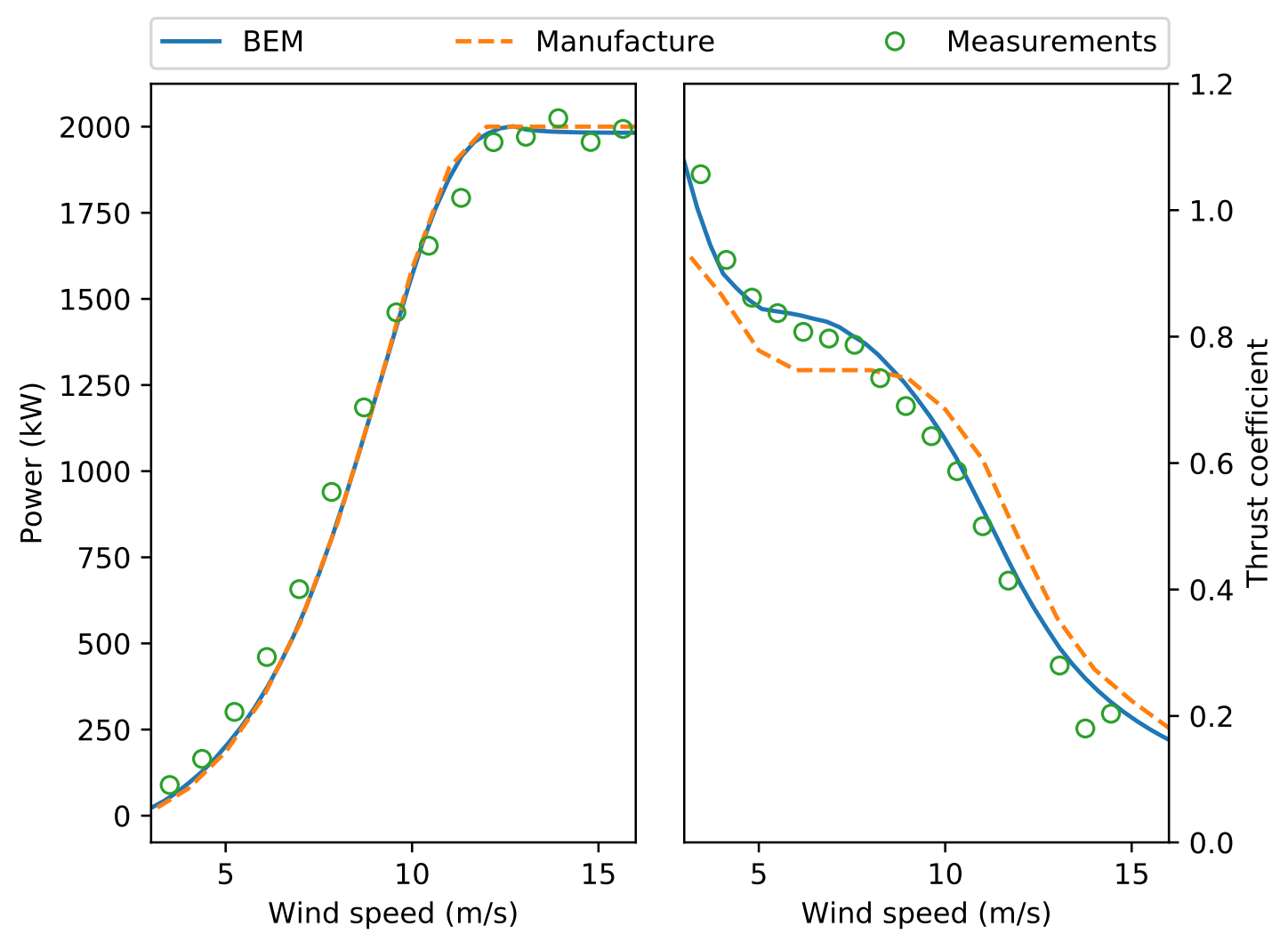

\subsection{The NTK500/41 Turbine Test Case}

Another test case is based on lidar measurements of wakes of a NTK500/41 turbine at the Risø Campus test site of DTU in Denmark [2] and is used to check whether the FullyRF similarity functions are site-dependent in wake simulations. The NTK500/41 turbine is a stall-regulated $500 \mathrm{~kW}$ wind turbine equipped with LM $19.1 \mathrm{~m}$ blades and its rotor speed is fixed at $27.1 \mathrm{rpm}$. A pulsed lidar mounted on a platform at the rear of the nacelle, pointed its laser downstream the turbine where a constant downhill slope of about $0.3 \%$ was observed. Inlet meteorological properties such as wind speed, wind direction, air temperature and atmospheric pressure were measured from a $57 \mathrm{~m}$ tall meteorological mast located upstream the turbine. Details of test cases are listed in Tab. 2. LES simulations of wakes of the NTK500/41 turbine [2] will be compared with the models proposed in this paper.

Table 2. Details of test cases.

\begin{tabular}{ccccc}
\hline Wind turbine & $D(\mathbf{m})$ & $H(\mathbf{m})$ & Measurements & Wake range \\
\hline H93-2.0MW & 93 & 67 & Mast measuremt & $1.25 D, 2.15 \mathrm{D}$ and $5 \mathrm{D}$ \\
NTK500/41 & 41 & 36 & Lidar scanning and LES simulations & $1 \mathrm{D}$ to $8 \mathrm{D}$ \\
\hline
\end{tabular}

For the NTK500/41 turbine, the power curve from BEM calculations has good agreements with the RANS computations in fully turbulence model [35] below $15 \mathrm{~m} / \mathrm{s}$, with LES computations [2] and measurements [35] below $10 \mathrm{~m} / \mathrm{s}$ (Fig. 4). The measured thrust coefficient from strain gauges measurements [36] is shown to have good agreements with the BEM computations and the LES computations above $6 \mathrm{~m} / \mathrm{s}$. The thrust coefficient applied in all simulations is from the BEM computations. 


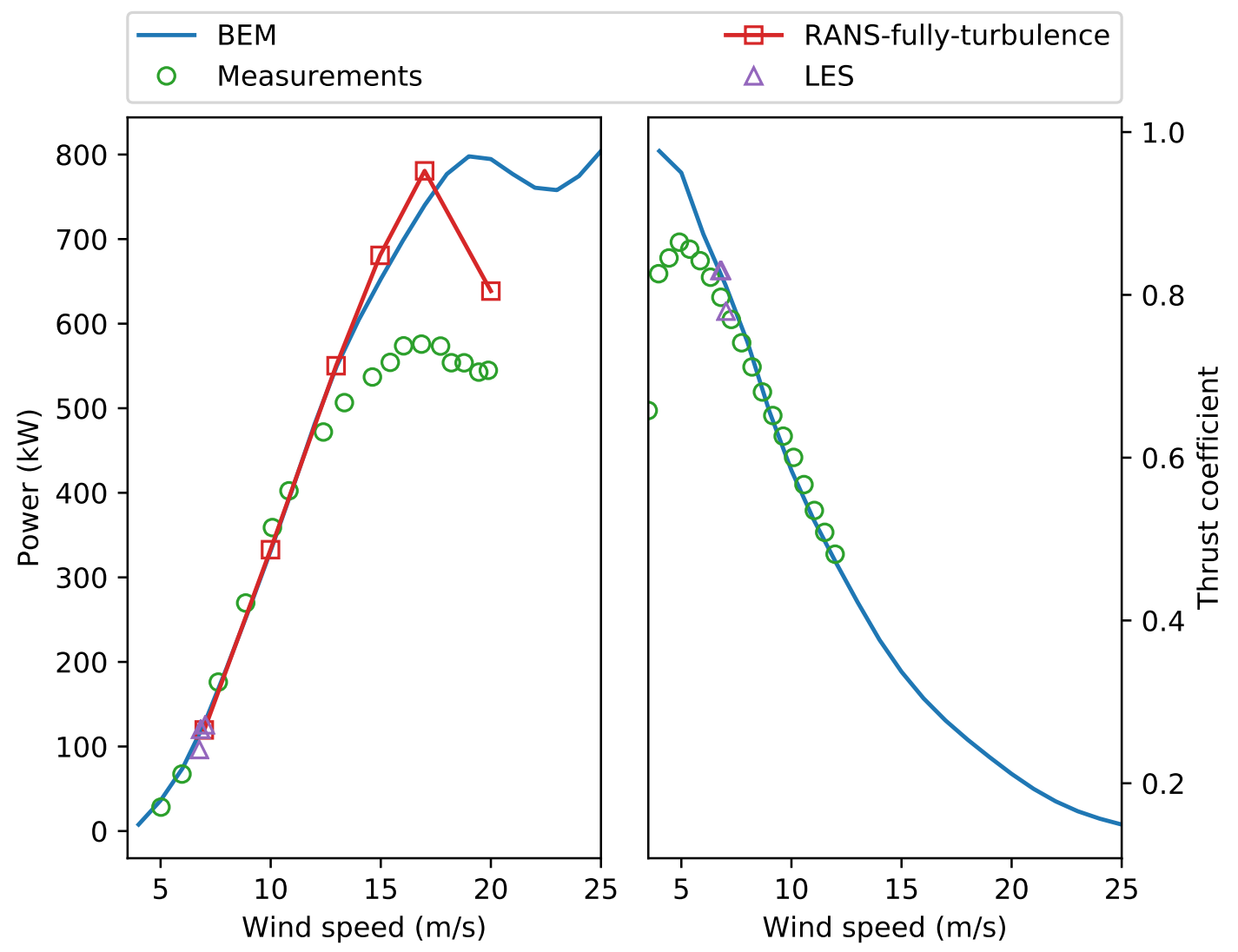

Figure 4. Performance curves of the NTK500/41 turbine.

\subsection{Computational Domain and Meshing}

The computational domain has a length of $20 D$, a width of $10 D$ and a height of $10 D$ (Fig. 5). All the AD models are implemented in the open source CFD software OpenFOAM [37]. The background mesh whose refinement level is 0 , consists of $200 \times 60 \times 60$ for the H93-2.0MW turbine and $100 \times 60 \times 60$ for the NTK500/41 turbine in length, width and height. The vertical grids are clustered near the ground and the first cell height above the ground is set to be $5.5 z_{0}$ [1]. The mesh is refined around the disk region to ensure 80 to 120 cells through the rotor diameter.

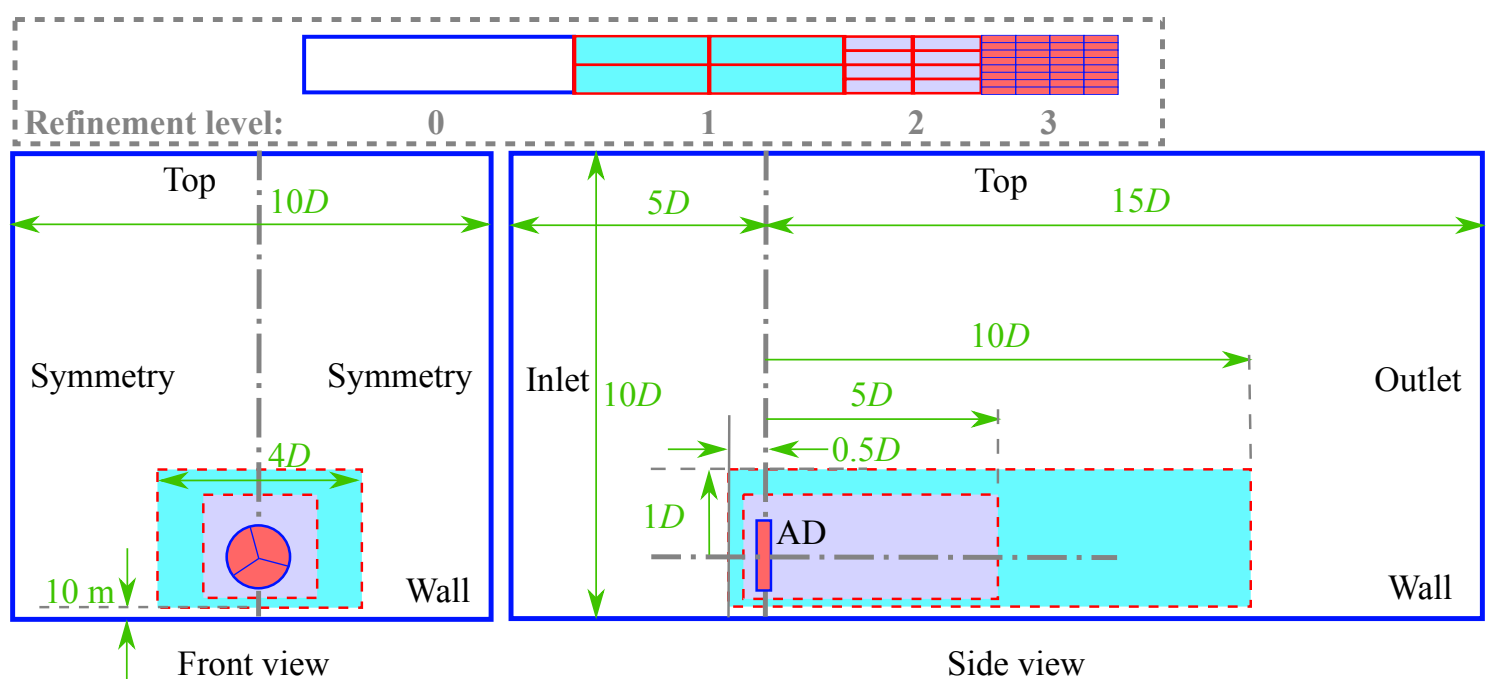

Figure 5. Compuational domain and meshing settings. 


\subsection{Boundary Conditions}

The boundary conditions consistent with similarity functions are applied to modeling the atmospheric boundary stratification. According to Han et al. [1], we apply the following inlet conditions in wake simulations:

$$
\begin{gathered}
U(z)=\int_{z_{0}}^{z} \frac{u_{*}}{\kappa z} \phi_{m}\left(\frac{z}{L}\right) \mathrm{d} z \\
\Theta(z)=\Theta_{0}+\int_{z_{0}}^{z} \frac{\theta_{*}}{\kappa z} \phi_{h}\left(\frac{z}{L}\right) \mathrm{d} z \\
\varepsilon=\frac{u_{*}^{3}}{\kappa z} \phi_{\varepsilon}\left(\frac{z}{L}\right) \\
k=\frac{u_{*}^{2}}{\sqrt{C_{\mu}}} \phi_{k}\left(\frac{z}{L}\right)
\end{gathered}
$$

where the roughness length $z_{0}$ approximates $0.05 \mathrm{~m}$ for the H93-2.0MW turbine [1] and $0.095 \mathrm{~m}$ for the NTK500/41 turbine [2]. The vertical profiles of wind speed and potential temperature are estimated in numerical integration. Zero gradients of $U, \Theta, \varepsilon, k$ applied at the outlet. $\varepsilon, k$ and vertical gradients of $U, \Theta$ are set to be fixed values on the top boundary [1]. And the turbulent law of wall presented in [1] is applied to the first layer of cells above the wall. The left and right sides of the computational domain are set to be symmetry.

The detail information of inlet conditions and resulting inlet veocity profiles are shown in Tab. 3 and in Fig. 6. The inlet velocity at hub is set to be $6 \mathrm{~m} / \mathrm{s}$ for the H93-2.0MW turbine and about 7 $\mathrm{m} / \mathrm{s}$ for the NTK500/41 turbine [2]. In the LES cases of the NTK500/41 turbine, the time-varying vertical structure of the whole atmosphere boundary layer was simulated based on the second GABLS experiment [38] to assess the mean and velocity profiles imposed at the inlet of the computational domain. In general, the surface-layer height decreases with atmospheric stability and thus limits the wind shear [39] under stable conditions. For very stable cases, the classical similarity functions strongly over-predicts the wind speed from $40 \mathrm{~m}$ upwards [12], which results in large discrepancy of velocity profile from measurements and based on the classical similarity functions and a larger shear compared with the measurements. The FullyRF similarity functions limit the wind shear under stable conditions and thus show good agreements with measurements. For the unstable test case, good agreements between the wind speed profile measured at the meteorological mast and the modeled inflow in LES, FullyRF model and Laan are observed, however the neutral wind profile is further off in LES. 


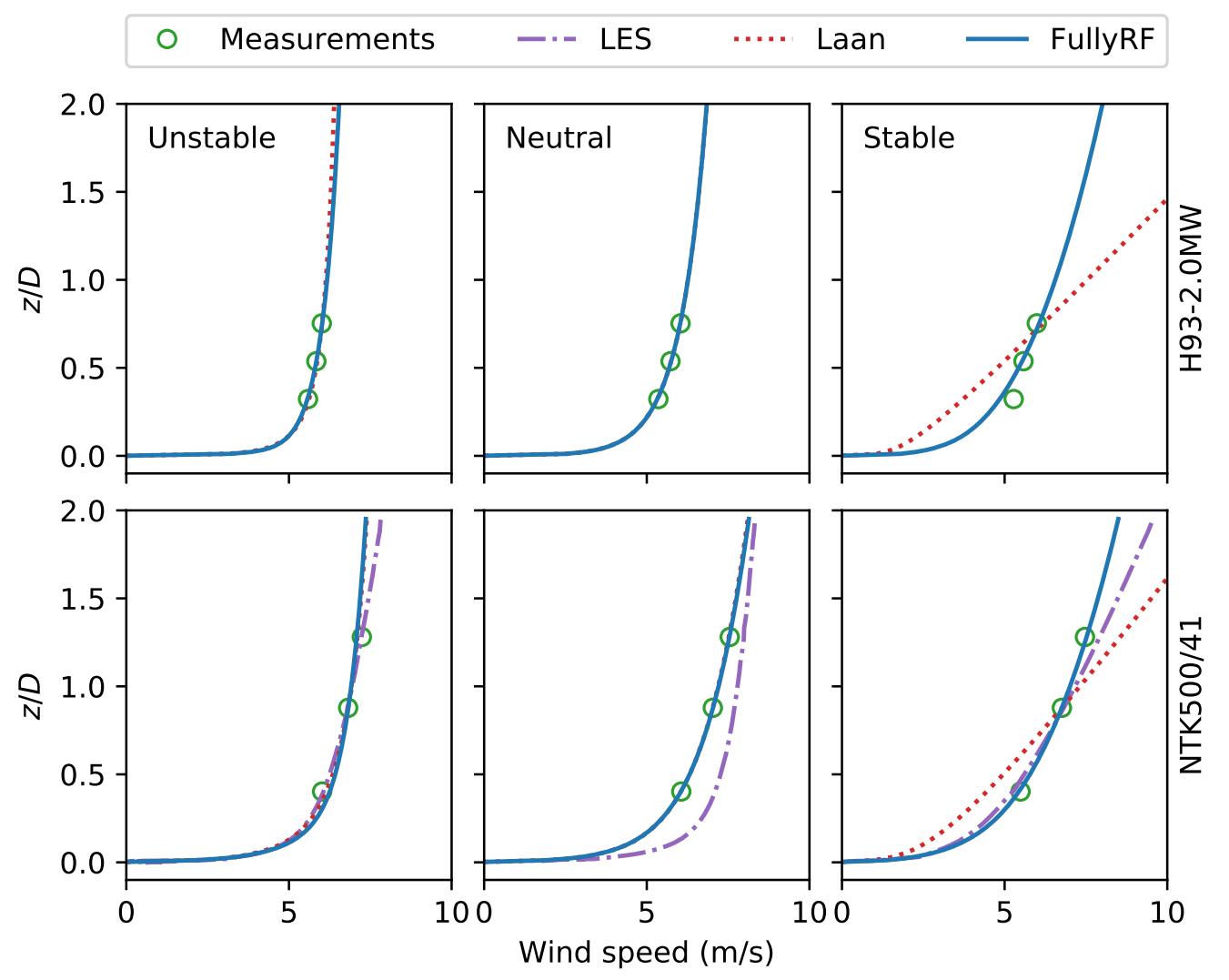

Figure 6. Inlet speed profiles.

Table 3. Details of inlet conditions.

\begin{tabular}{|c|c|c|c|c|c|c|}
\hline \multirow{2}{*}{ Stability } & \multirow{2}{*}{ Model } & \multicolumn{2}{|c|}{ H93-2.0MW } & \multicolumn{3}{|c|}{ NTK500/41 } \\
\hline & & $L(\mathrm{~m})$ & $u_{*}(\mathrm{~m} / \mathrm{s})$ & $L(\mathrm{~m})$ & $u_{*}(\mathrm{~m} / \mathrm{s})$ & $U_{\text {hub }}(\mathrm{m} / \mathrm{s})$ \\
\hline Unstable & All & -30 & 0.425 & -84.8 & 0.525 & 6.82 \\
\hline Neutral & All & $\infty$ & 0.333 & 1677.7 & 0.464 & 7.03 \\
\hline Stable & Laan & 30 & 0.131 & 29.0 & 0.223 & 6.76 \\
\hline
\end{tabular}

\section{Results and Discussion}

\subsection{Wakes of the H93-2.0MW turbines}

Fig. 7 to Fig. 9 show the wake profile at $1.45 D, 2.15 D$ and $5 D$ under different stability conditions. The significant effects of atmoshperic stability that can be observed from measurements are: (1) Wake deficits increase with atmospheric stability. Higher wake deficit are observed under stable or neutral conditions than unstable conditions: the maximum wake deficit at $70 \mathrm{~m}$ height in $1.45 \mathrm{D}$ downstream the turbine in the unstable case rises from 0.5 to 0.6 in the neutral or stable case. (2) Wake recovers much slower under neutral and stable conditions than under unstable conditions. The normalized wake velocity at $70 \mathrm{~m}$ height under unstable, neutral and stable conditions rises to 0.73 , 0.64 and 0.62 , respectively. This is mainly because turbulence is depressed under stable conditions and the momentum and energy exchanges between the wake and the atmosphere are thus weaken. (3) Double-bell near-wake shape at $70 \mathrm{~m}$ height, probably due to a lower energy extraction around the blade root [2], are observed for neutral and stable cases at 1.45D. A local minimum deficit occurs in the wake center and the difference between this minimum value and the local maximum deficit around the wake center ranges from 0.1 (neutral) to 0.2 (stable). (4) The double-bell near-wake shape is slightly 
asymmetric and this asymmetric can be enhanced by atmospheric stability. More deficit is observed on the left side of the turbine when looking at the turbine from downwind [40]. The asymmetry wake is also observed by Menke et al. [7].

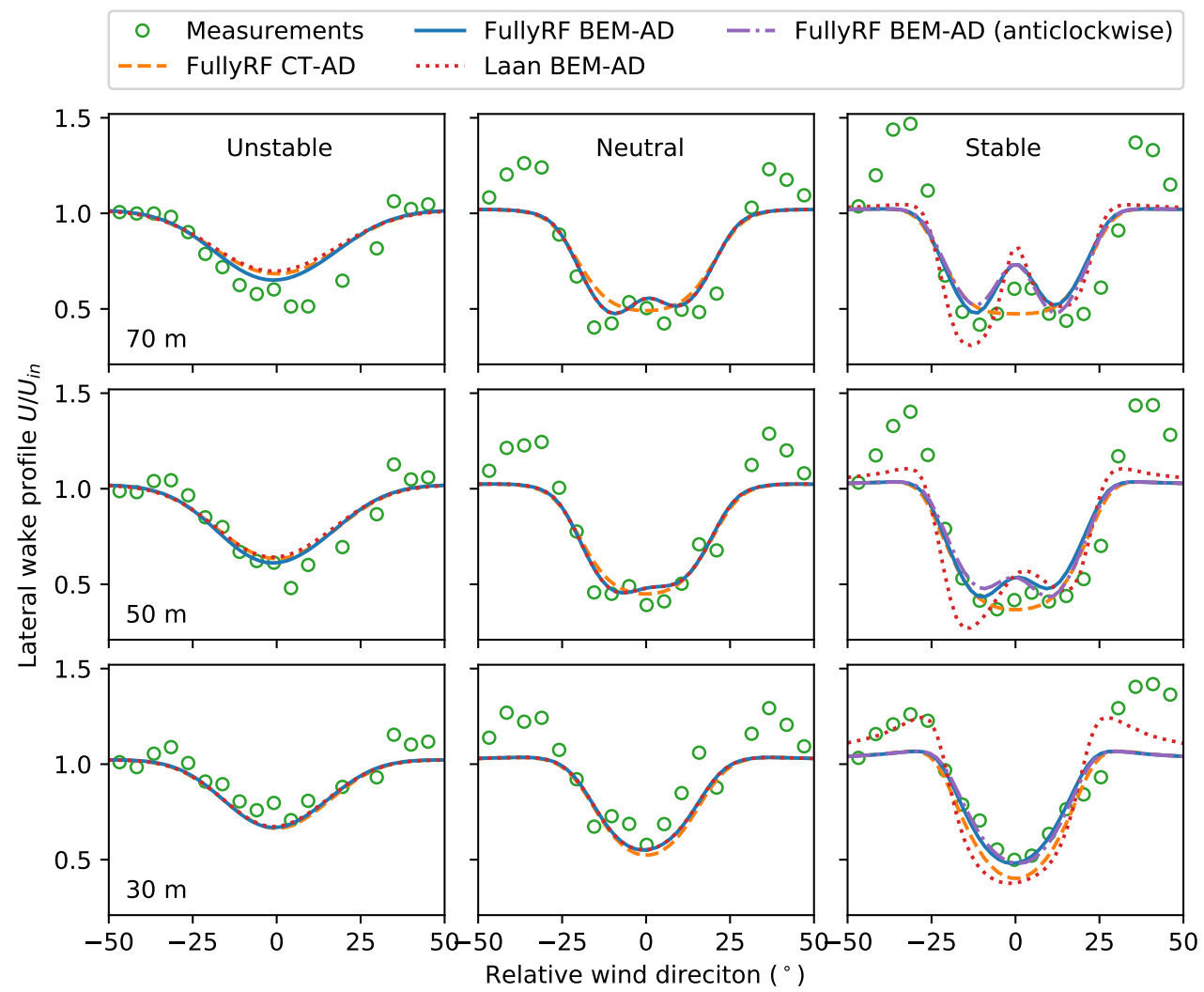

Figure 7. Wake profile at $1.45 \mathrm{D}$ and $U_{\text {in }}$ is the mean inlet velocity.

The FullyRF BEM-AD model has a good agreement with measurements except for multiple wake cases. At $1.45 D$, the inlet wind speed at M1 could be affected of wakes of \#12 and \#15, which results in a significant discrepancy between the measurements and simulations around the relative wind direction $\pm 30^{\circ}$ especially under neutral and stable conditions, see Appendix A for the definition of the relative wind direction in a wake. In the stable case, the FullyRF CT-AD model fails to predict the double-bell near-wake shape and tends to overestimate the near-wake deficit at lower part of wakes (30 $\mathrm{m}$ at 1.45, $50 \mathrm{~m}$ and $30 \mathrm{~m}$ at 2.15D) while the Laan BEM-AD model yields an enhanced asymmetric double-bell near-wake shape and overestimates the wake deficit despite of the longitudinal distance to the rotor. Since the similarity functions of the Laan model and the FullyRF model are very similar under unstable conditions, there are no major differences on the wake deficit predictions between the three AD models. 


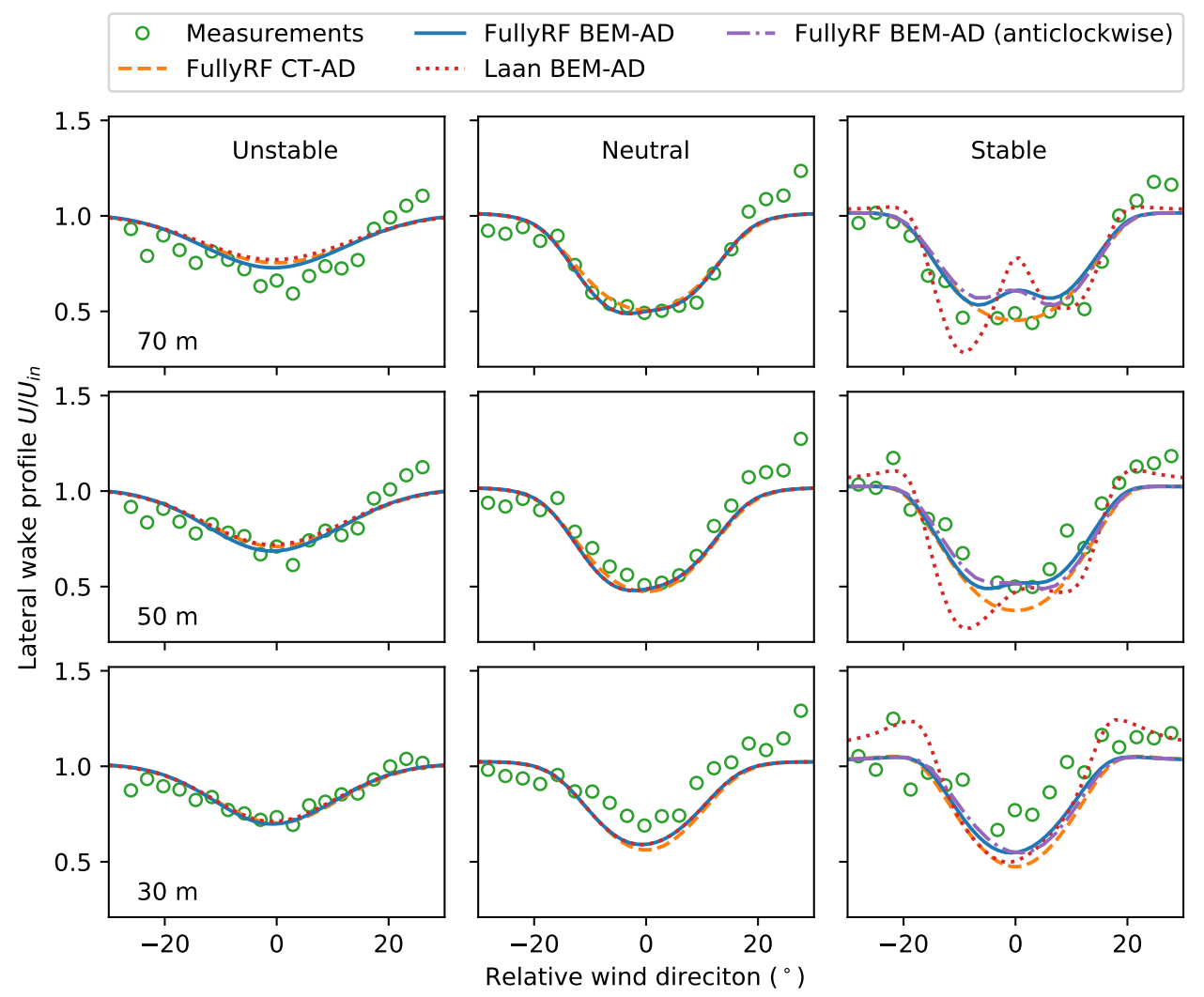

Figure 8. Wake profile at $2.15 \mathrm{D}$

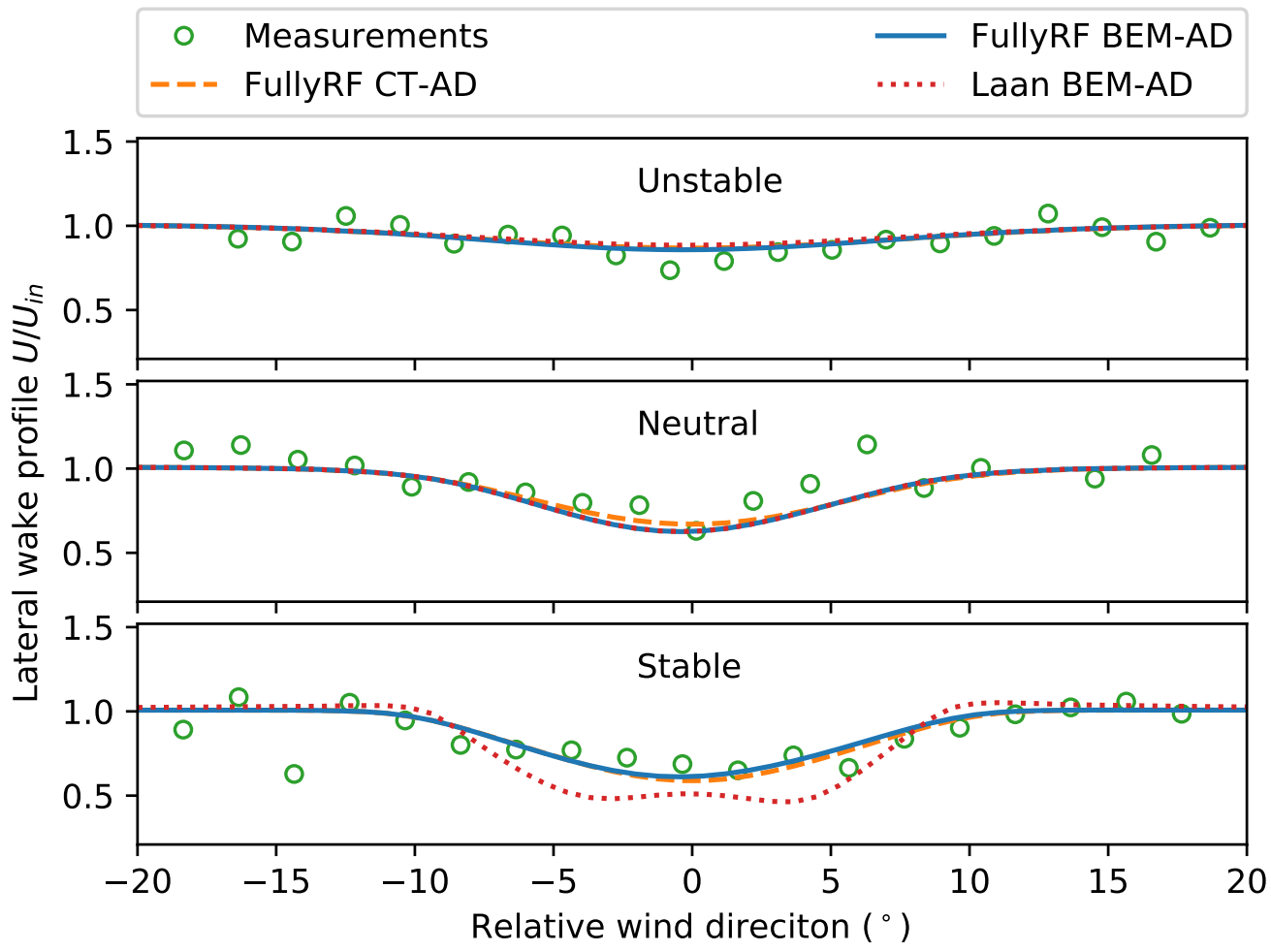

Figure 9. Wake profile at $5 \mathrm{D}$ 
Both measurements and simulations show that the double bell is not symmetric to the wake center although it is slight in measurements. Churchfield et al. [40] relates this phenomenon to the asymmetric vertical profile due to wind shear and the wake rotation that counters the blade rotation. For the clockwise-rotation turbine, the wake rotation is anticlockwise and transfers low-speed flow from below to the left side of the wake and high-speed flow from above to the right when looking at the turbine from downwind. In this study, the high wind shear of the Laan BEM-AD model tends to enhance this asymmetry and countering the blade rotation to anticlockwise does reverses the local maximum of wake deficit from the left side to the right side of the wake.

The asymmetric double-bell near-wake shape is also clearly observed for the turbulence intensity increase $\Delta I$ in the wind turbine wakes at $1.45 D$ under neutral and stable conditions. The proposed model predicts the double-bell near-wake shape and underestimates the maximum increase of turbulence intensity. However, this underestimation decreases with atmospheric stability and wake recovery. In cases without the double-bell near-wake shape, the increase of turbulence intensity predicted by the AD models show good agreements with measurements.

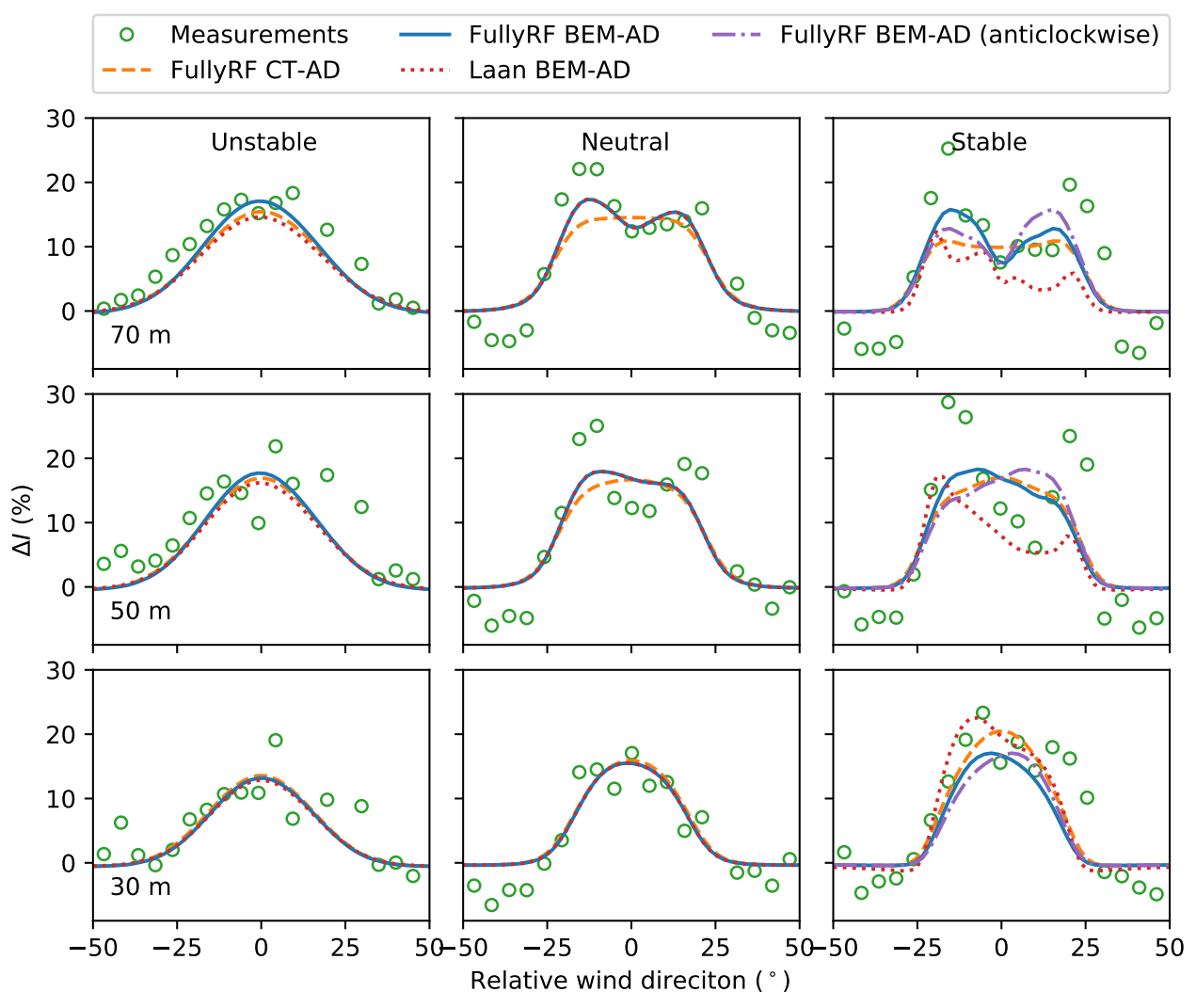

Figure 10. $\Delta I$ at $1.45 D$ 


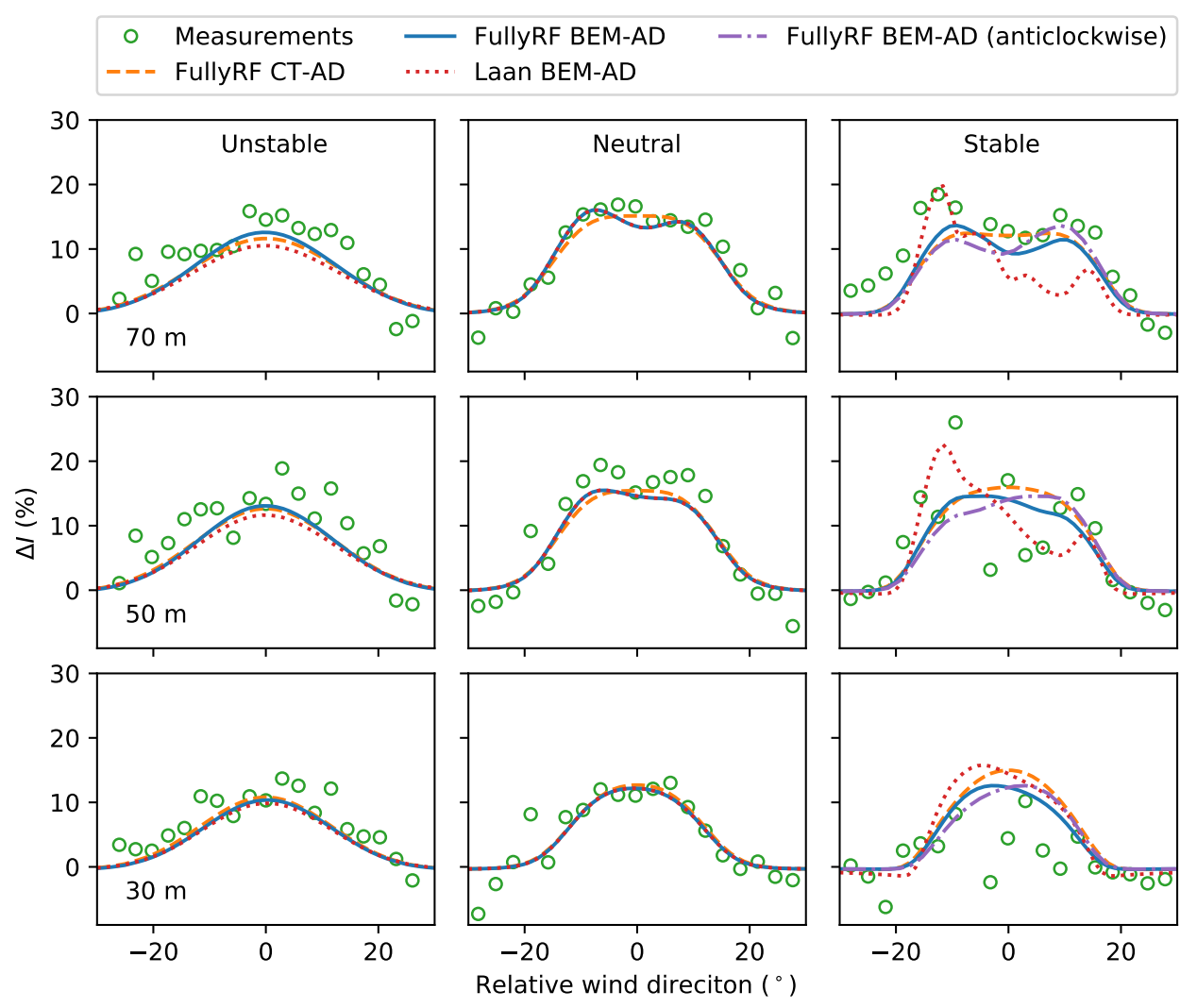

Figure 11. $\Delta I$ at $2.15 \mathrm{D}$

274 Due to the absence of the turbulence intensity data at wind turbines, the turbulence intensity at $275 \# 12$ is approximated by measurements at M1 for the wake of wt12 to M1 and is set to $15 \%, 9 \%$, and $2766 \%$ for unstable, neutral and stable conditions, respectively. At $5 D, \Delta I$ predicted by the FullyRF AD 277 models have good agreements with measurements while being overestimated by the Laan BEM-AD ${ }_{278}$ model (Fig. 12). 


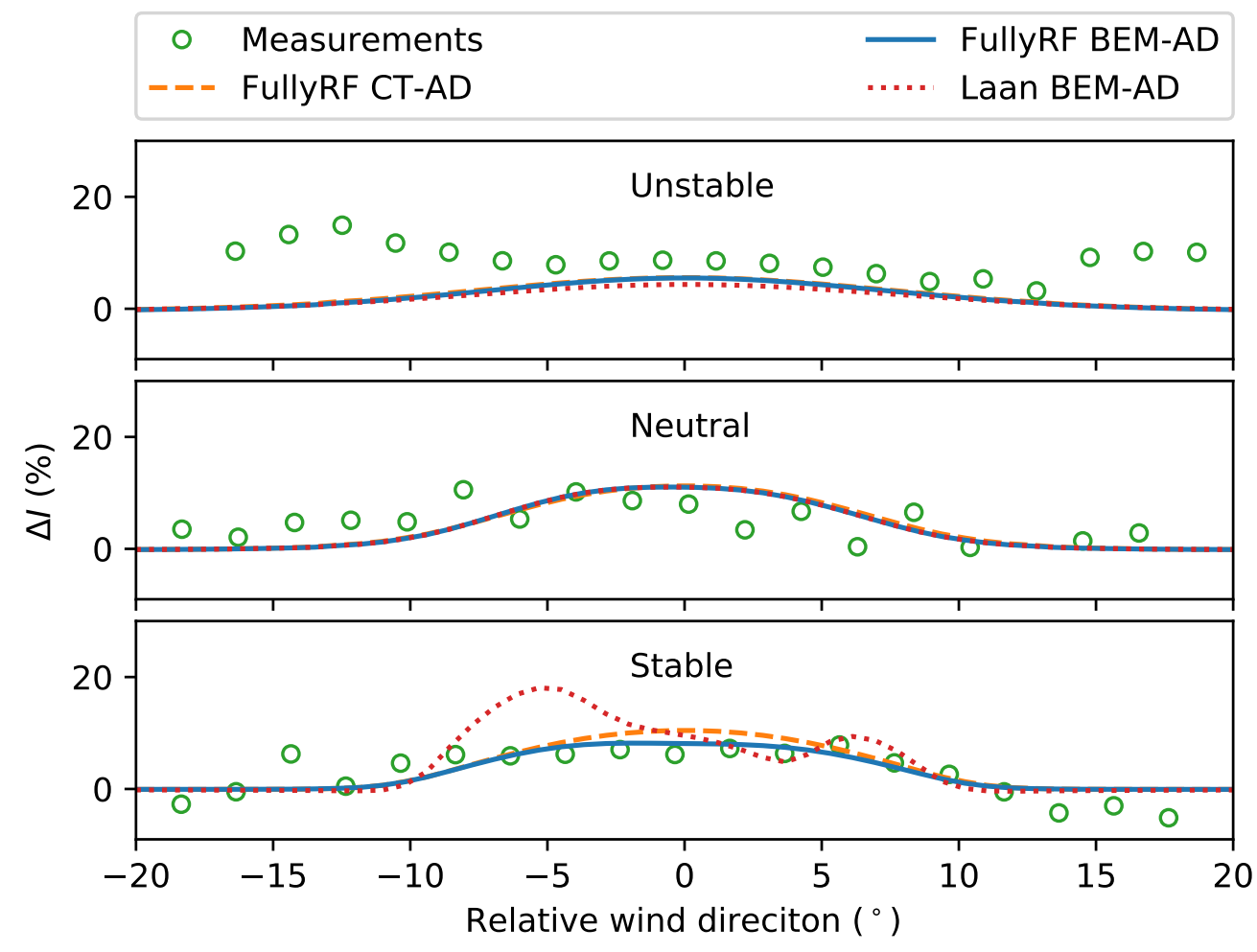

Figure 12. $\Delta I$ at $5 D$

\subsection{Wakes of the NTK500/41 Turbine}

Fig. 13 shows the comparisons of normalized velocity contour in wakes under stable conditions. The simulation in LES show larger deficits in near wake and a slightly faster recovery wake than that in the proposed model, while the Laan BEM-AD model predicts a slower recovery wake with much larger deficit. For a given reference wind speed $U_{\text {hub }}$ and a fixed roughness length $z_{0}$, higher wind shear in the Laan model results in a smaller friction speed $u_{*}$ and a lower turbulence intensity than the proposed model and thus weakens the wake recovery. 


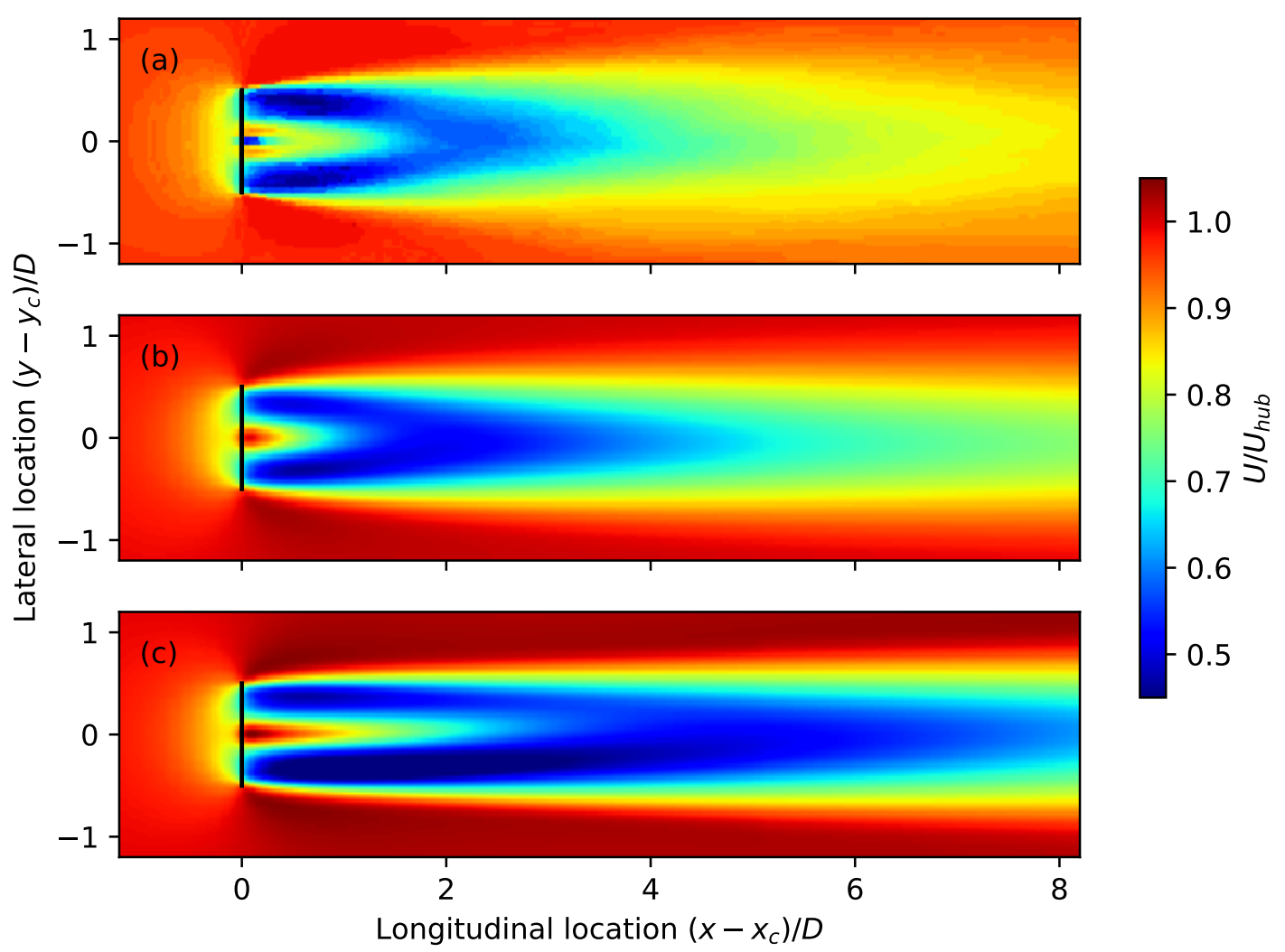

Figure 13. Normalized velocity distribution at hub height in the stable case where $\left(x_{c}, y_{c}, z_{c}\right)$ is the center point of the disk.

As compared with measurements, the proposed model shows better performance than other AD approaches in RANS and is comparable to the LES approach (Fig. 14). For the longitudinal distance above $2 D$, the Laan BEM-AD model overestimates the wake deficits in both vertical and lateral directions. Since the wake tends to follow terrain under very stable conditions, the wind speed for the longitudinal distance above $5 D$ at the hub height is actually the value of $5.5 \mathrm{~m}$ above the wake center. Considering such an effect, the proposed model shows a good agreement with measurements at $5 \mathrm{D}$. Due to the absence of wake measured data above $5 D$, it is difficult to distinguish which is better for wake predictions between the proposed model and the LES approach. The slower wake recovery over $5 D$ predicted by the proposed model in Fig. 13 as compared to the LES approach, should be studied in further research. 


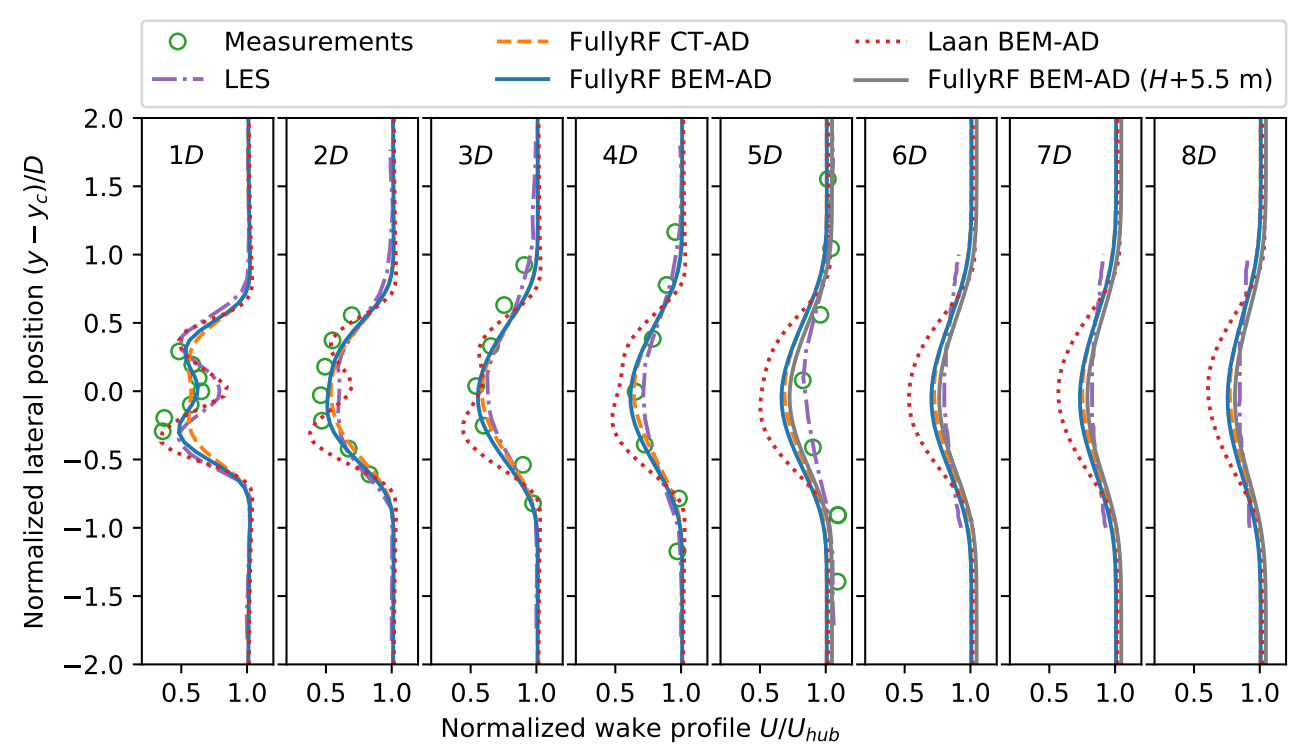

Figure 14. Wake deficit at hub height in the stable case.

In the stable case, the proposed model underestimates wake deficits above the hub height at $1 D$ and $2 D$ while overestimating wake deficits below the hub height at $5 D$ (Fig. 15). There are no major differences between the vertical wake profiles predicted by the FullyRF CT-AD model and the proposed model above 2D. However, the Laan BEM-AD model significantly overestimates wake deficits at $4 D$ and 5D. As the LES model [2] applies slip wall conditions at the bottom of the domain without considering the roughness effect, large discrepancies of wind profile are observed near the ground.

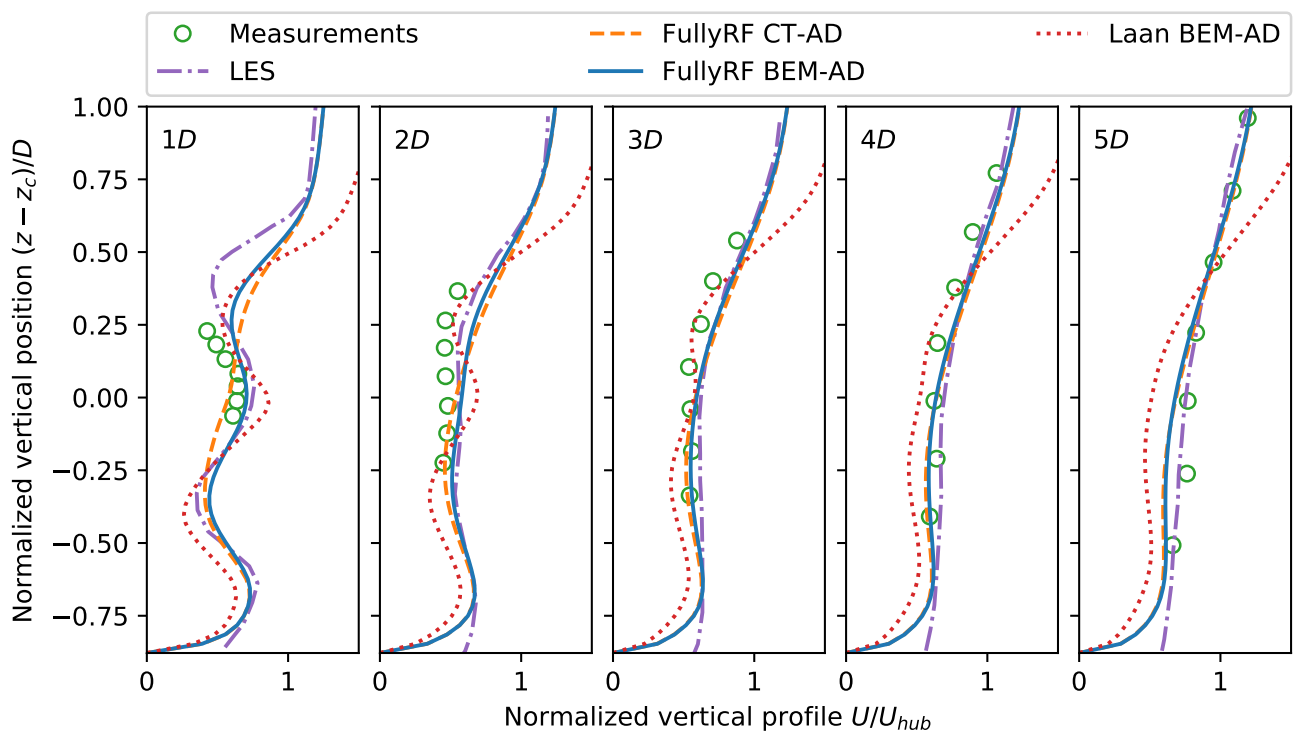

Figure 15. Vertical wake deficit profile in the stable case.

In the neutral case, the double-bell near-wake shape is only observed at $1 D$ and captured by the proposed model and the LES approach (Fig. 16). Measurements show good agreements with predictions of the proposed model at $1 D$ to $3 D$. Since the wake also follows the slope slightly under neutral conditions [2], the proposed model show a slightly decay recovery wake for the longitudinal distance above $4 \mathrm{D}$. In Fig. 17, effects of slope are considered by rising the measured data slightly, and the predictions of the proposed model tends to approach the measurements. 


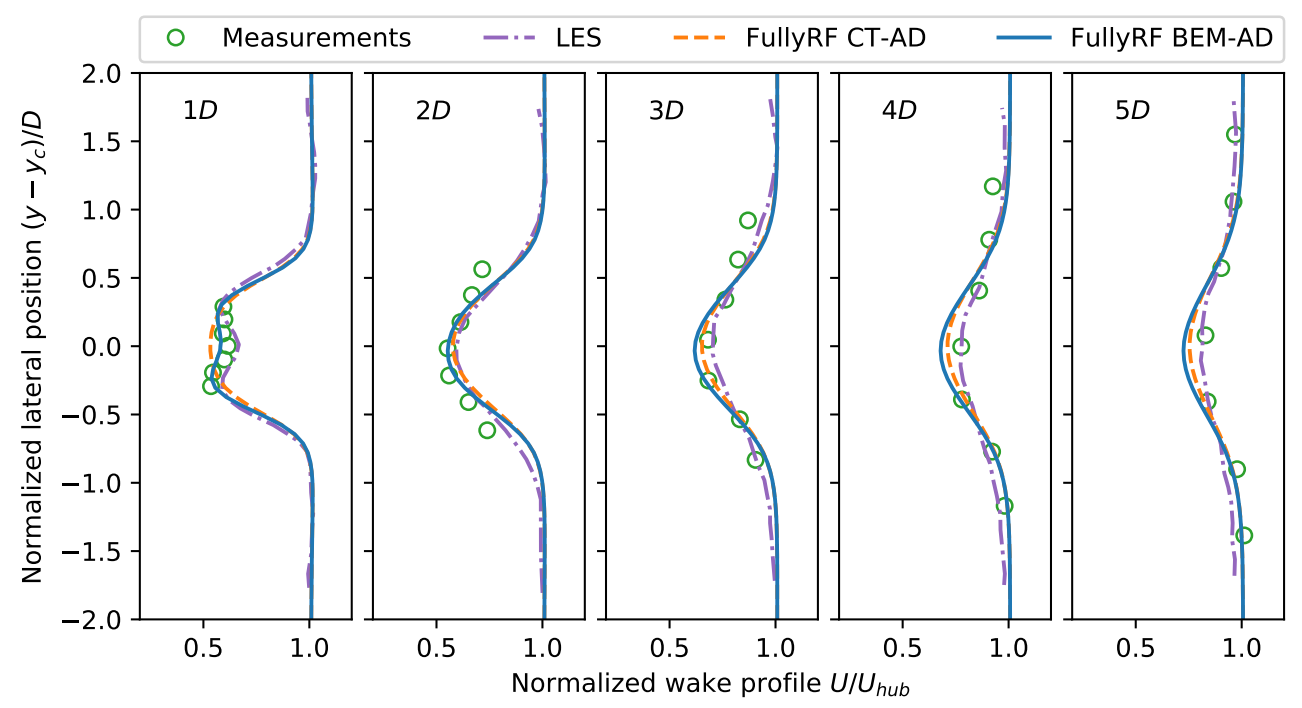

Figure 16. Wake deficit at hub height in the neutral case.

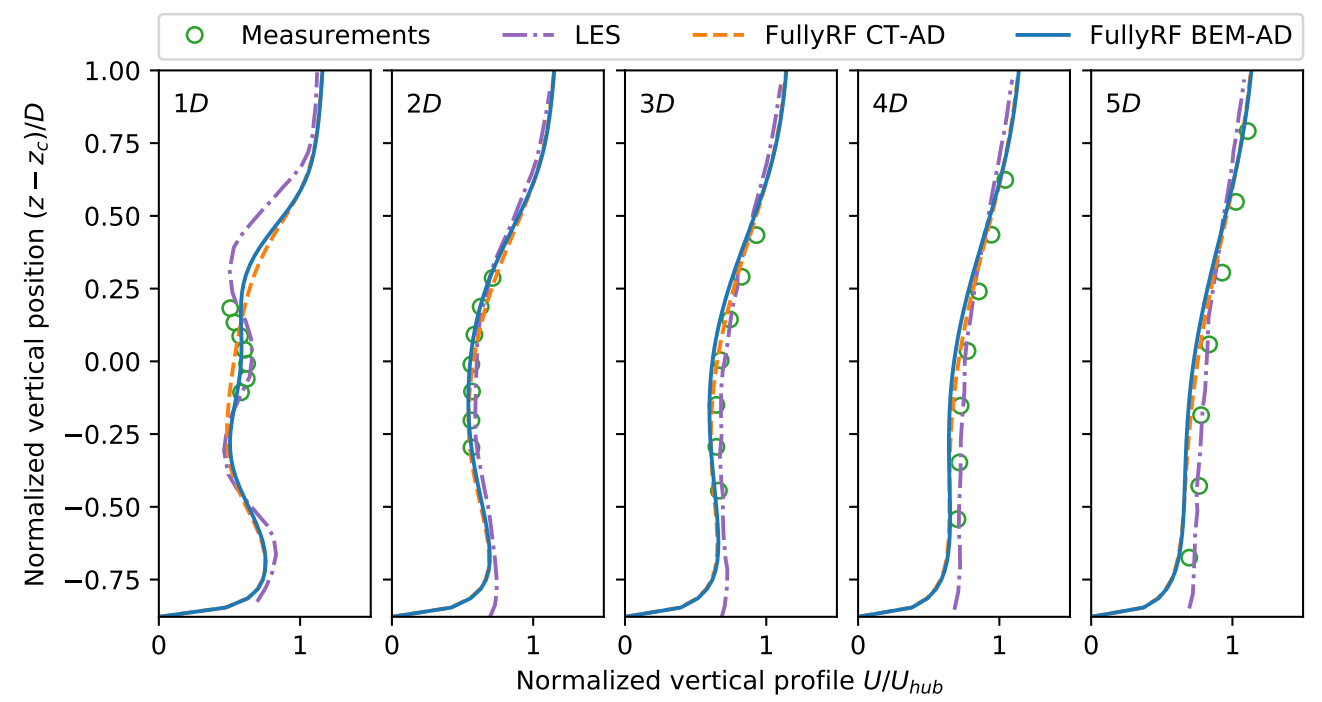

Figure 17. Vertical wake deficit profile in the neutral case.

Fig. 18 shows the comparisons of normalized wind speed contour in wakes under unstable conditions from simulations using different approaches. The simulation in LES show larger deficits in near wake and a slightly slower wake recovery than that predicted by the proposed model or the Laan BEM-AD model. 


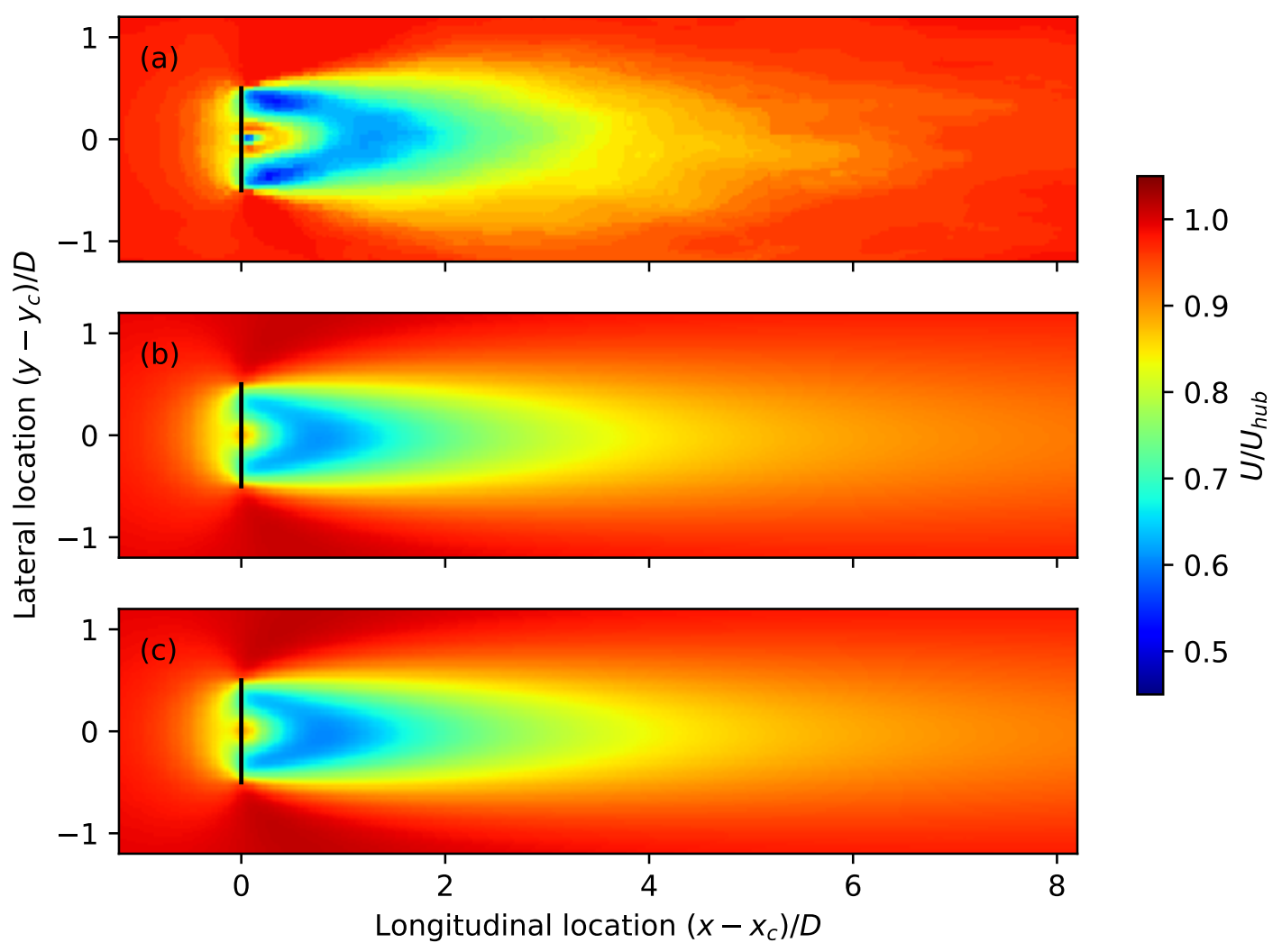

Figure 18. Normalized velocity distribution at hub height in the unstable case.

All the models in this paper, fail in predicting near wake under unstable conditions (Fig. 19 and Fig. 20): the measurements show a larger deficit than simulations at $1 D$ and at $2 D$. However, for the longitudinal distance above $2 D$, all the simulations show good agreements with the measurements. Compared with the good agreements between measurements using anemometers in the H93-2.0MW cases and predictions of the proposed model, the near-wake measurements using lidars could introduce much uncertainties due to wake meandering under unstable conditions and the terrain effects [2].

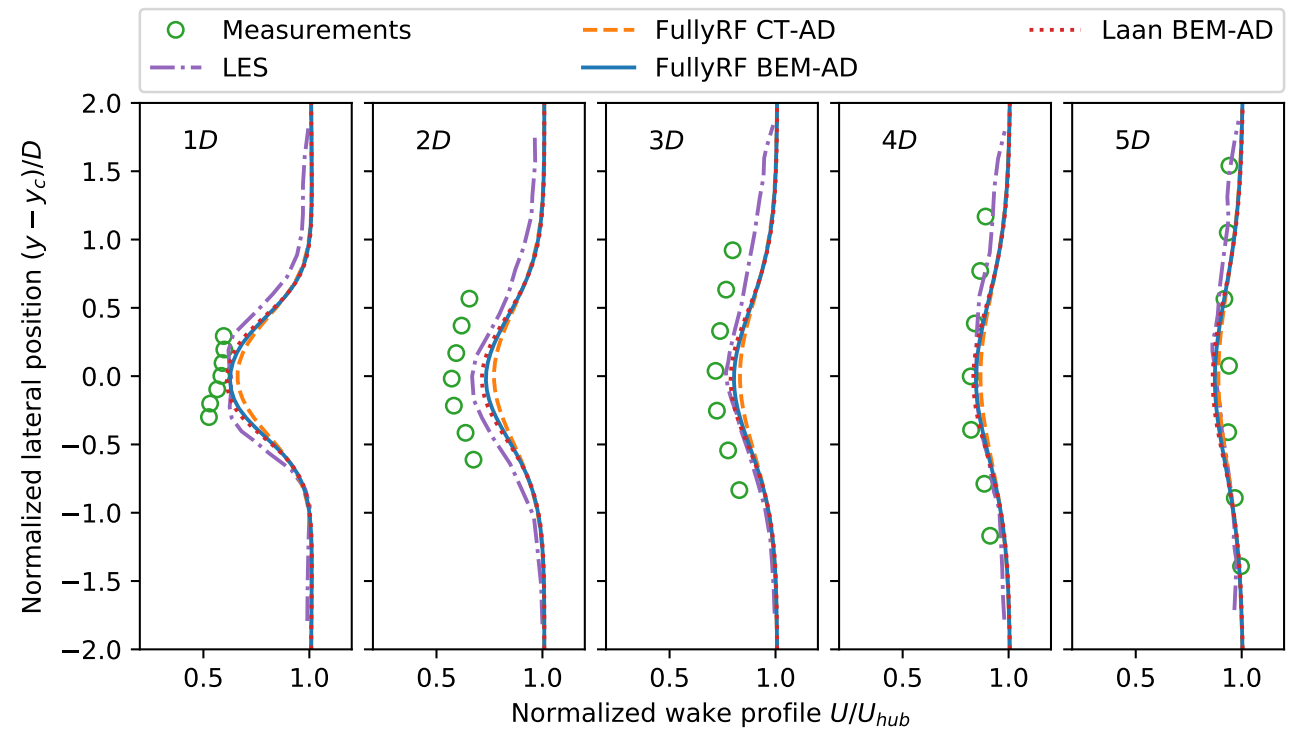

Figure 19. Wake deficit at hub height in the unstable case. 


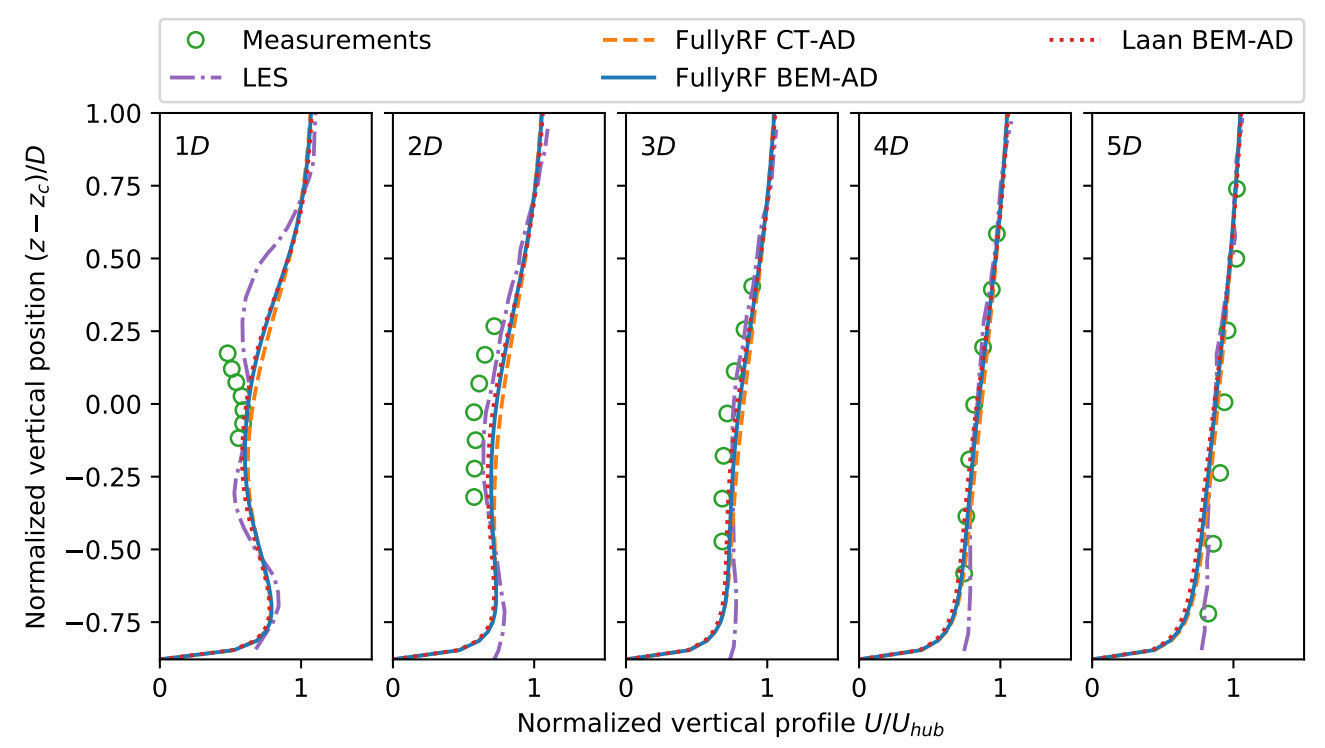

Figure 20. Vertical wake deficit profile in the unstable case.

\section{Conclusions}

In the present paper, the AD modeling of wind turbine wakes in RANS under varying atmospheric stability conditions are studied by focusing the effects of the distributed blade force based on BEM and the effects of the limited wind shear in the FullyRF model.

Wake simulations of two types of turbines under different stability conditions are carried out and compared with measurements from cup anemometers and a lidar. According to measurements, the wake tends to be enhanced and recovers slower in stable cases than in unstable cases, as a consequence of the depressed turbulence process. Asymmetric double-bell wake profiles are observed in near wake, especially for stable cases, which is probably caused by the lower extraction of energy at the rotor hub location. This asymmetry wake could be strengthened by high wind shear under stable conditions and converted by countering the blade rotation.

By distributing the blade force through the rotor, momentum effects of rotor to the atmospheric boundary layer are simulated in more detail way than the uniformly distributed blade force applied by the CT-AD method. This enables the proposed model to capture the double-bell near-wake shape. For the cases without the double-bell shape, predictions of the FullyRF models show a fair agreement with measurements. However, the Laan BEM-AD model significantly overestimates wake deficits and predicts a fairly slow recovery wake as compared to measurements, which is mainly due to the higher wind shear than measured data and much lower turbulence intensity than the FullyRF models. For cases that applied a lidar to measure the wake, predictions of the LES approach show a good agreement with measurements except for near wake under unstable conditions. And the LES approach predicts that the wake recovers slightly slower under unstable conditions and faster under stable conditions, as compared to the proposed model. Due to the fair agreement between the predictions of wakes in the proposed model and the measurements and the much lower computational resources than the LES approach, the proposed model is suitable for wind energy engineering applications.

Author Contributions: Data curation, Xing Xing Han; Formal analysis, Xing Xing Han; Methodology, Xing Xing Han; Project administration, De You Liu; Software, Xing Xing Han and Lin Min Li; Supervision, De You Liu; Validation, Xing Xing Han and Fei Fei Xue; Visualization, Xing Xing Han; Writing - original draft, Xing Xing Han; Writing - review editing, Chang Xu and Wen Zhong Shen.

Funding: This research was funded by the Ministry of Science and Technology of China grant number 2014DFG62530, the Danish Energy Agency grant number 64013-0405, the Joint Funds of the National Natural Science Foundation of China grant number U1865101, Jiangsu provincial science and Technology Department grant number BZ2018007.

Conflicts of Interest: The authors declare no conflict of interest. 


\author{
Abbreviations \\ The following abbreviations are used in this paper: \\ CFD Computational Fluid Dynamics \\ ASL Atmospheric Surface Layer \\ MOST Moin-Obukhov Similarity Theory \\ BEM Blade Element Theory \\ RANS Reynolds-averaged Navier-Stokes Equations \\ LES Large Eddy Simulation \\ AD Actuator Disk \\ AL Actuator Line
}

\title{
Appendix A Relative Wind Direction in Wake
}

The relative direction in a wake is defined as the angle between the inlet wind direction and the line from the upstream wind turbine to the mast. When looking at the turbine from downwind, this relative direction is negative at the left side and positive at the right side of the wake.

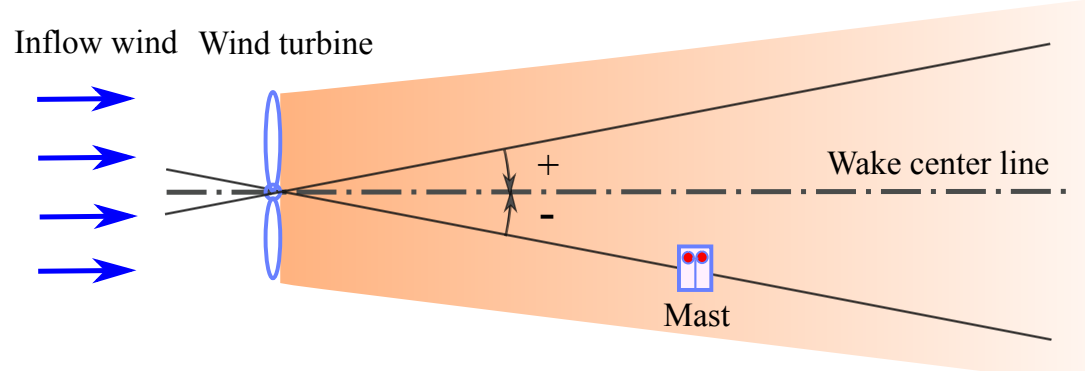

Figure A1. Definition of the relative direction.

\section{References}

1. Han, X.X.; Liu, D.Y.; Xu, C.; Shen, W.Z. Similarity functions and a new $k-\varepsilon$ closure for predicting stratified atmospheric surface layer flows in complex terrain. Renewable energy, Under Review.

2. Machefaux, E.; Larsen, G.C.; Koblitz, T.; Troldborg, N.; Kelly, M.C.; Chougule, A.; Hansen, K.S.; Rodrigo, J.S. An experimental and numerical study of the atmospheric stability impact on wind turbine wakes. Wind Energy 2016, 19, 1785-1805.

3. Chamorro, L.P.; Porté-Agel, F. Effects of Thermal Stability and Incoming Boundary-Layer Flow Characteristics on Wind-Turbine Wakes: A Wind-Tunnel Study. Boundary-Layer Meteorology 2010, 136, 515-533. doi:10.1007/s10546-010-9512-1.

4. Zhang, W.; Markfort, C.D.; Porté-Agel, F. Wind-turbine wakes in a convective boundary layer: A wind-tunnel study. Boundary-layer meteorology 2013, 146, 161-179.

5. Hancock, P.; Zhang, S.; Pascheke, F.; Hayden, P. Wind tunnel simulation of a wind turbine wake in neutral, stable and unstable wind flow. Journal of Physics: Conference Series. IOP Publishing, 2014, Vol. 555, p. 012047.

6. Iungo, G.V.; Porté-Agel, F. Volumetric lidar scanning of wind turbine wakes under convective and neutral atmospheric stability regimes. Journal of Atmospheric and Oceanic Technology 2014, 31, 2035-2048.

7. Menke, R.; Vasiljević, N.; Hansen, K.S.; Hahmann, A.N.; Mann, J. Does the wind turbine wake follow the topography? A multi-lidar study in complex terrain. Wind Energy Science 2018, 3, 681-691.

8. Wu, Y.T.; Porté-Agel, F. Atmospheric turbulence effects on wind-turbine wakes: An LES study. energies 2012, 5, 5340-5362.

9. Xie, S.; Archer, C.L. A numerical study of wind-turbine wakes for three atmospheric stability conditions. Boundary-Layer Meteorology 2017, 165, 87-112.

10. Wu, Y.T.; Porté-Agel, F. Large-eddy simulation of wind-turbine wakes: evaluation of turbine parametrisations. Boundary-layer meteorology 2011, 138, 345-366. 
11. Churchfield, M.; Lee, S.; Moriarty, P. Overview of the simulator for wind farm application (SOWFA). National Renewable Energy Laboratory 2012.

12. Peña, A.; Gryning, S.E.; Mann, J. On the length-scale of the wind profile. Quarterly Journal of the Royal Meteorological Society 2010, 136, 2119-2131.

13. Porté-Agel, F.; Wu, Y.T.; Lu, H.; Conzemius, R.J. Large-eddy simulation of atmospheric boundary layer flow through wind turbines and wind farms. Journal of Wind Engineering and Industrial Aerodynamics 2011, 99, 154-168.

14. Moens, M.; Duponcheel, M.; Winckelmans, G.; Chatelain, P. An actuator disk method with tip-loss correction based on local effective upstream velocities. Wind Energy 2018, 21, 766-782.

15. El Kasmi, A.; Masson, C. An extended k-e model for turbulent flow through horizontal-axis wind turbines. Journal of Wind Engineering and Industrial Aerodynamics 2008, 96, 103-122.

16. Van der Laan, M.; Hansen, K.S.; Sørensen, N.N.; Réthoré, P.E. Predicting wind farm wake interaction with RANS: an investigation of the Coriolis force. Journal of Physics: Conference Series. IOP Publishing, 2015, Vol. 625, p. 012026.

17. Xu, C.; Han, X.; Wang, X.; Liu, D.; Zheng, Y.; Shen, W.Z.; Zhang, M. Study of wind turbine wake modeling based on a modified actuator disk model and extended k- $\varepsilon$ turbulence model. Zhongguo Dianji Gongcheng Xиеbao 2015, 35, 1954-1961.

18. Hennen, J.; Kenjereš, S. Contribution to improved eddy-viscosity modeling of the wind turbine-to-wake interactions. International Journal of Heat and Fluid Flow 2017, 68, 319-336.

19. Taylor, G. Wake measurements on the Nibe turbines in Denmark; Energy Technology Support Unit, 1990.

20. Cal, R.B.; Lebrón, J.; Castillo, L.; Kang, H.S.; Meneveau, C. Experimental study of the horizontally averaged flow structure in a model wind-turbine array boundary layer. Journal of Renewable and Sustainable Energy 2010, 2, 013106.

21. van der Laan, M.P.; Kelly, M.C.; Sørensen, N.N. A new k-epsilon model consistent with Monin-Obukhov similarity theory. Wind Energy 2017, 20, 479-489.

22. Alinot, C.; Masson, C. $k$-varepsilon Model for the Atmospheric Boundary Layer Under Various Thermal Stratifications. Journal of Solar Energy Engineering 2005, 127, 438-443.

23. Van Der Avoird, E.; Duynkerke, P.G. Turbulence in a katabatic flow. Boundary-Layer Meteorology 1999, 92,37-63.

24. Koblitz, T.; Sørensen, N.N.; Bechmann, A.; Sogachev, A. CFD Modeling of Non-Neutral Atmospheric Boundary Layer Conditions; DTU Wind Energy, 2013.

25. Shen, W.Z.; Mikkelsen, R.; Sørensen, J.N.; Bak, C. Tip Loss Corrections for Wind Turbine Computations. Wind Energy 2005, 8, 457-475. doi:10.1002/we.153.

26. Drela, M. XFOIL: An analysis and design system for low Reynolds number airfoils. In Low Reynolds number aerodynamics; Springer, 1989; pp. 1-12.

27. Du, Z.; Selig, M. A 3-D stall-delay model for horizontal axis wind turbine performance prediction. 1998 ASME Wind Energy Symposium, 1998, p. 21.

28. Shen, W.Z.; Zhu, W.J.; Sørensen, J.N. Actuator Line/Navier-Stokes Computations for the MEXICO Rotor: Comparison with Detailed Measurements. Wind Energy 2012, 15, 811-825. doi:10.1002/we.510.

29. Glauert, H. Airplane propellers. In Aerodynamic theory; Springer, 1935; pp. 169-360.

30. Foken, T. 50 years of the Monin-Obukhov similarity theory. Boundary-Layer Meteorology 2006, 119, 431-447. 31. Businger, J.A.; Wyngaard, J.C.; Izumi, Y.; Bradley, E.F. Flux-profile relationships in the atmospheric surface layer. Journal of the atmospheric Sciences 1971, 28, 181-189.

32. Dyer, A. A review of flux-profile relationships. Boundary-Layer Meteorology 1974, 7, 363-372.

33. Grachev, A.A.; Andreas, E.L.; Fairall, C.W.; Guest, P.S.; Persson, P.O.G. The critical Richardson number and limits of applicability of local similarity theory in the stable boundary layer. Boundary-layer meteorology 2013, 147, 51-82.

34. Han, X.; Liu, D.; Xu, C.; Shen, W.Z. Atmospheric stability and topography effects on wind turbine performance and wake properties in complex terrain. Renewable energy 2018, 126, 640-651.

35. Johansen, J.; Sørensen, N.N. Aerofoil characteristics from 3D CFD rotor computations. Wind Energy: An International Journal for Progress and Applications in Wind Power Conversion Technology 2004, 7, 283-294.

36. Vignaroli, A. UniTTe-MC1-Nordtank Measurement Campaign (Turbine and Met Masts) 2016. 
436

437

37. Weller, H.G.; Tabor, G.; Jasak, H.; Fureby, C. A tensorial approach to computational continuum mechanics using object-oriented techniques. Computers in physics 1998, 12, 620-631.

38. Svensson, G.; Holtslag, A.; Kumar, V.; Mauritsen, T.; Steeneveld, G.; Angevine, W.; Bazile, E.; Beljaars, A.; De Bruijn, E.; Cheng, A.; others. Evaluation of the diurnal cycle in the atmospheric boundary layer over land as represented by a variety of single-column models: the second GABLS experiment. Boundary-Layer Meteorology 2011, 140, 177-206.

39. Optis, M.; Monahan, A.; Bosveld, F.C. Limitations and breakdown of Monin-Obukhov similarity theory for wind profile extrapolation under stable stratification. Wind Energy 2016, 19, 1053-1072.

40. Churchfield, M.J.; Lee, S.; Michalakes, J.; Moriarty, P. A numerical study of the effects of atmospheric and wake turbulence on wind turbine dynamics. Journal of Turbulence 2012, 13. 